\title{
破产终端值影响下保险公司最优分红、注资 和溢额再保险策略
}

\author{
姚定俊 ${ }^{1}$, 汪荣明 ${ }^{2}$, 徐林 ${ }^{3}$ \\ 1. 南京财经大学金融学院, 南京 210023 ; \\ 2. 华东师范大学统计学院, 上海 $200241 ;$ \\ 3. 安徽师范大学数学计算机科学学院, 芜湖 241003 \\ E-mail: yaodingjun@sina.cn,wang_rongm@yahoo.com,xulinahnu@gmail.com
}

收稿日期: 2014-08-27；接受日期: 2016-10-13；网络出版日期: 2017-03-22；＊通信作者

国家自然科学基金 (批准号: 71671082, 71471081, 11571113, 11231005, 11101205 和 11201006)、教育部人文社会科学研究 (批准 号: 15YJC910008)、上海市优秀学术带头人计划 (批准号: 14XD1401600)、高等学校学科创新引智计划 (批准号: B14019) 和江苏 省高校自然科学研究 (批准号: 15KJB110009) 资助项目

摘要 本文假设保险公司可通过分红、注资和购买溢额再保险三种方式动态地控制盈余过程.同时控 制过程会消耗交易费用：再保险合同是“不便宜的”; 分红需要纳税; 而注资需要比例注资成本和固定 注资成本。在最大化公司价值目标下, 本文给出了最优的控制策略, 并分析了交易费用和任意破产终 端值的影响. 结果表明, 当交易费用较低且破产终端值相对较小时, 注资可能最优, 应当按照 Barrier 策略进行分红; 当公司盈余增加时, 应当减少再保险购买量.

关键词 分红 注资 溢额再保险 终端值 交易费用 最优策略

MSC (2010) 主题分类 $62 \mathrm{P} 05,97 \mathrm{M} 30,93 \mathrm{E} 20$

\section{1 引言}

从公司金融的角度来看, 公司价值 (或股东利益) 可定义为未来红利的期望现值. 金融数学中分红 策略研究可以追溯到文献 [1], 其核心问题是在最大化公司价值的目标下制定最优的分红策略. 此后, 许多学者在各种风险模型下研究了最优分红策略问题并取得了丰硕的成果. 另外, 再保险在保险公司 经营中扮演着重要角色. 保险公司往往通过再保险合约将承保风险部分转嫁给再保险公司并支付一定 的保费, 从而达到降低自身经营风险的目的. 由于比例再保险和溢额再保险在实践中最为常见, 因此, 这两种再保险参与下的最优分红问题一直是学者们关注的热点, 包括文献 [2-8] 等部分重要文献.

在实践中, 公司破产时往往存在破产终端值 (如残值和清算成本等), 它的存在影响到公司价值. 基 于此, 一些学者开始在最优分红和再保险问题中考虑破产终端值的影响. 相应地, 公司价值被定义为 破产前分红与破产终端值的期望现值之和. 例如, 文献 $[9,10]$ 分别在比例再保险和溢额再保险假设下 研究了破产终端值对最优分红和再保险策略的影响. 这两篇文献均假设再保险是 “便宜的”, 即再保险 人向原保险人收取的费率等于原保险人向投保人收取的费率. 那么从理论上讲, 原保险人可以将承保

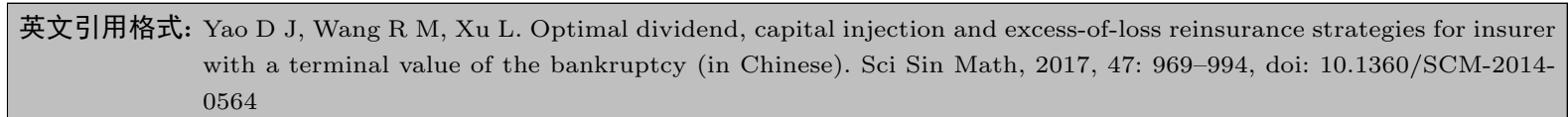

(c) 2017 《中国科学》杂志社

www.scichina.com mathcn.scichina.com 
风险全部转移给再保险人而无需支付任何额外费用, 无需注资也可使破产永不发生. 此时, 如果破产 终端值是负数, 那么保险公司就可采取这种措施避免破产清算. 显然, 这与事实不符. 文献 $[11,12]$ 在 其他模型下也遇到了类似的问题. 最近, 文献 [13] 在最优分红问题中引入了 “不便宜的” 比例再保险, 即假设再保险人向原保险人收取的费率大于原保险人向投保人收取的费率. 此时原保险人无法通过转 移全部风险的方法保持盈余过程的非负性, 那么即使破产终端值为负数时原保险人也可能被迫选择破 产. 文献 [13] 在任意破产终端值假设下研究了最优分红和比例再保险问题. 该文献的研究提供了很好 的思路, 但该文献没有考虑交易费用和注资的可能性.

除了上述分红和再保险外，注资也是保险公司重要的经营手段．当考虑注资时，可将公司价值定 义为破产前分红与注资期望现值之差, 管理者需要在最大化公司价值目标下制定最优的分红、注资和 再保险策略. 为了更加贴近市场, 学者们进一步考虑分红和注资过程中交易费用的影响, 使得问题更加 复杂. 交易费用影响下的最优分红和注资策略研究可参见文献 [14-19] 等, 最优分红、注资和再保险策 略研究可参见文献 [20-23] 等.

在以上研究的基础上, 本文关注一个最优的分红、注资和溢额再保险策略问题. 为使模型更具实 际意义, 我们假设再保险是 “不便宜的”; 分红需要纳税; 而注资需要消耗比例的融资成本和固定的融 资成本; 一旦保险公司破产, 股东就要对破产终端值 $P \in \mathbb{R}$ 负责. 我们采用一个与文献 [9] 相同的运行 函数度量保险公司价值, 它综合考虑了分红、注资、破产时刻、破产终端值和交易费用的影响. 在最大 化公司价值的目标下, 我们分 14 种不同的情形给出了最优的分红、注资和溢额再保险策略.

本文结构如下: 第 2 节用扩散模型模拟保险公司的盈余过程, 并引入最优控制策略问题. 第 3 节 给出最优控制策略问题对应的 HJB (Hamilton-Jacobi-Bellman) 方程以及验证性定理. 根据再保险成 本的不同, 我们分别在第 4 和 5 节中求解 HJB 方程从而得到了对应的值函数 (即最大的运行函数), 并构造了最优的动态控制策略. 第 6 节总结全文.

\section{2 风险模型和最优控制问题}

记 $\left(\Omega, \mathcal{F}, \mathcal{F}_{t}, \mathrm{P}\right)$ 为概率空间, 其中 $\mathcal{F}_{t}$ 表示到 $t$ 时刻的信息流, 它满足通常条件. 为便于表述, 我们 从经典的复合 Poisson 模型开始. 该模型用参数为 $\lambda$ 的 Poisson 过程 $N_{t}$ 表示到 $t$ 时刻为止的理赔次 数; 用 $Y_{i}$ 表示第 $i$ 次理赔额, 且随机理赔额 $\left\{Y_{i}, i=1,2, \ldots\right\}$ 独立同分布, 其累积分布函数为 $F(\cdot)$. 考 虑到可保条件, 我们假设理赔分布的支撑有限, 即 $m:=\sup \{x: F(x)<1\}<\infty$. 记 $\mu_{m}=\mathrm{E}\left(Y_{1}\right)<\infty$ 和 $\nu_{m}=\mathrm{E}\left(Y_{1}^{2}\right)<\infty$. 该模型假设下保险公司在 $t$ 时刻的盈余可表示为

$$
U_{t}=x+c t-\sum_{i=1}^{N_{t}} Y_{i}
$$

这里 $U_{0-}=x \geqslant 0$ 是初始盈余, $c \geqslant 0$ 是单位时间内收取的保费. 假设保险公司采用参数为 $\theta_{1}>0$ 的 期望值保费原理进行定价, 那么,

$$
c=\left(1+\theta_{1}\right) \mathrm{E}\left(\sum_{i=1}^{N_{1}} Y_{i}\right)=\left(1+\theta_{1}\right) \lambda \mu_{m},
$$

这里 $\mathrm{E}$ 表示求期望值. 进一步假设保险公司可以购买再保险. 我们用可测函数 $R(\cdot)$ 表示再保险合约 安排, 它对概率空间上任意非负随机变量都有 $0 \leqslant R(Y) \leqslant Y$ 成立. 对任意潜在的理赔额 $Y$, 保险公 司自留风险为 $R(Y)$, 向再保险公司分出的风险为 $Y-R(Y)$. 假设再保险公司同样采用期望值原理向 
原保险公司收取保费, 但是使用一个较大的安全负荷 $\theta_{2} \in\left(\theta_{1}, \infty\right)$, 即再保险是 “不便宜的”. 到时间 1 为止, 保险公司分出风险为 $\sum_{k=1}^{N_{1}}\left(Y_{k}-R\left(Y_{k}\right)\right)$. 相应地, 向再保险公司支付的保费为

$$
c^{R}=\left(1+\theta_{2}\right) \mathrm{E}\left[\sum_{k=1}^{N_{1}}\left(Y_{k}-R\left(Y_{k}\right)\right)\right]=\left(1+\theta_{2}\right) \lambda\left[\mu_{m}-\mathrm{E}(R(Y))\right]
$$

于是, 再保险合约 $R$ 下原保险公司的盈余过程可表示为

$$
U_{t}^{R}=x+\left(c-c^{R}\right) t-\sum_{i=1}^{N(t)} R\left(Y_{i}\right) .
$$

为了问题的简化, 我们用期望和方差均与 (2.4) 相同的扩散过程 $\left\{X_{t}^{R}, t \geqslant 0\right\}$ 去近似保险公司的盈余过 程 (参见文献 [24]). 当保险公司业务量较大时, 这样的近似处理较合理. 此时, $X_{t}^{R}$ 满足如下随机过程:

$$
X_{t}^{R}=x+\left[\left(\theta_{1}-\theta_{2}\right) \lambda \nu_{m}+\theta_{2} \lambda \mathrm{E}\left(R\left(Y_{1}\right)\right)\right] t+\sqrt{\lambda \mathrm{E}\left[\left(R\left(Y_{k}\right)\right)^{2}\right]} B_{t},
$$

这里 $X_{0-}^{R}=x \geqslant 0,\left\{B_{t}, t \geqslant 0\right\}$ 是信息流 $\left\{\mathcal{F}_{t}\right\}$ 上的标准 Brown 运动. 记 $\mathcal{F}^{B}=\left\{\mathcal{F}_{t}^{B}, t \geqslant 0\right\}$ 为该 Brown 运动生成的自然流

因为本文注 2.1 中所述原因, 我们取 $R(Y)=Y \wedge a$, 即 $R(\cdot)$ 定义了一个溢额再保险合同, 这里 $a \in[0, m]$ 是保险公司的风险自留水平. 相应地, 对任意理赔额 $Y_{i}$, 原保险公司承担 $Y_{i} \wedge a$, 而再保险公 司承担 $\left(Y_{i}-a\right)_{+}=Y_{i}-Y_{i} \wedge a$. 记

$$
\begin{aligned}
& \mu(a):=\mathrm{E}\left(Y_{1} \wedge a\right)=\int_{0}^{a} \bar{F}(y) d y, \\
& \nu(a):=\mathrm{E}\left(Y_{1} \wedge a\right)^{2}=\int_{0}^{a} 2 y \bar{F}(y) d y,
\end{aligned}
$$

其中 $\bar{F}(y)=\mathrm{P}\left(Y_{1}>y\right)=1-F(y)$. 显然, $\mu(a)$ 和 $\nu(a)$ 在区间 $[0, m]$ 上关于变量 $a$ 递增. 在区间 $[m, \infty)$ 上它们都是常数, 分别记为 $\mu_{m}=\mu(m)$ 和 $\nu_{m}=\nu(m)$. 本文将要用到如下重要性质:

$$
\nu_{m}=\int_{0}^{m} 2 y \bar{F}(y) d y \leqslant 2 m \int_{0}^{m} \bar{F}(y) d y=2 m \mu_{m} .
$$

考虑到溢额再保险策略取决于风险自留水平 $a$, 我们将 (2.3) 改写为

$$
c^{a}=\left(1+\theta_{2}\right) \mathrm{E}\left(\sum_{i=1}^{N_{1}}\left(Y_{i}-Y_{i} \wedge a\right)\right)=\left(1+\theta_{2}\right) \lambda\left(\mu_{m}-\mu(a)\right) .
$$

相应地, 随机过程 (2.5) 改写为

$$
X_{t}^{a}=x+\left[\left(\theta_{1}-\theta_{2}\right) \lambda \mu_{m}+\theta_{2} \lambda \mu(a)\right] t+\sqrt{\lambda \nu(a)} B_{t},
$$

这里 $X_{0-}^{a}=x \geqslant 0$.

假设保险公司可以动态调整风险自留水平 $a \in[0, m]$, 那么, $\left\{a_{t}, t \geqslant 0\right\}$ 就可以描述动态的再保险 策略. 另外, 令 $D_{t}$ 表示到 $t$ 时刻为止的累积分红量. 用一列停时 $\left\{\tau_{i}, i=1,2, \ldots\right\}$ 表示历次注资时刻, 用一列非负随机变量 $\left\{\eta_{i}, i=1,2, \ldots\right\}$ 表示历次注资额, 那么, $R_{t}=\sum_{i=1}^{\infty} I_{\left\{\tau_{i} \leqslant t\right\}} \eta_{i}$ 可以表示到 $t$ 时刻 
为止保险公司的累积注资额. 保险公司整体决策可以用 $\pi=\left(a^{\pi} ; D^{\pi} ; R^{\pi}\right)$ 刻画. 在策略 $\pi$ 控制下盈余 过程可表示为

$$
X_{t}^{\pi}=x+\int_{0}^{t}\left[\left(\theta_{1}-\theta_{2}\right) \lambda \mu_{m}+\theta_{2} \lambda \mu\left(a_{s}^{\pi}\right)\right] d s+\int_{0}^{t} \sqrt{\lambda \nu\left(a_{s}^{\pi}\right)} d B_{s}+\sum_{i=1}^{\infty} I_{\left\{\tau_{i}^{\pi} \leqslant t\right\}} \eta_{i}^{\pi}-D_{t}^{\pi},
$$

这里 $X_{0-}^{\pi}=x \geqslant 0$. 下面给出保险公司可采取的容许策略定义.

定义 2.1 我们称

$$
\pi=\left(a^{\pi} ; D^{\pi} ; R^{\pi}\right)=\left(a^{\pi} ; D^{\pi} ; \tau_{1}^{\pi}, \ldots, \tau_{i}^{\pi}, \ldots ; \eta_{1}^{\pi}, \ldots, \eta_{i}^{\pi}, \ldots\right)
$$

为容许策略, 如果它满足如下条件:

(i) $a^{\pi}=\left\{a_{t}^{\pi}, t \geqslant 0\right\}$ 是 $\mathcal{F}^{B}$ 上的适应过程, 且 $0 \leqslant a_{t}^{\pi} \leqslant m$;

(ii) $D^{\pi}=\left\{D_{t}^{\pi}, t \geqslant 0\right\}$ 是 $\mathcal{F}^{B}$ 上递增的适应过程, 满足 $D_{0-}^{\pi}=0$ 和 $\Delta D_{t}^{\pi}=D_{t}^{\pi}-D_{t-}^{\pi} \leqslant X_{t-}^{\pi}$;

(iii) $\left\{\tau_{i}^{\pi}, i=1,2, \ldots\right\}$ 是 $\mathcal{F}^{B}$ 上一列递增的停时, 且 $0 \leqslant \tau_{1}^{\pi}<\cdots<\tau_{i}^{\pi}<\cdots$, a.s.;

(iv) $\eta_{i}^{\pi} \geqslant 0(i=1,2, \ldots)$ 关于 $\mathcal{F}_{\tau_{i}^{\pi}}^{B}$ 可测;

(v) $\mathrm{P}\left(\lim _{i \rightarrow \infty} \tau_{i}^{\pi}<t\right)=0, \forall t>0$.

条件 (ii) 表明管理者不可以借贷发放红利, 条件 (v) 要求有限时间内最多可有限次注资. 我们 将容许策略生成的空间记为 $\Pi$. 对任意 $\pi \in \Pi$, 我们把破产时刻定义盈余首次为负数的时刻, 记为 $T^{\pi}=\inf \left\{t \geqslant 0: X_{t}^{\pi}<0\right\}$. 假设一旦破产发生, 则公司需要进行破产清算并对破产终端值 $P \in \mathbb{R}$ 负 责. 当 $P \geqslant 0$ 时, 它可以被看作公司破产残值; 如果 $P<0$, 则可以理解为股东需要支付的破产清算成 本. 为了贴近金融市场, 我们考虑分红和注资过程中的交易费用. 如果资金 $d$ 元被用于股东分红, 那么 股东获得的税后红利为 $\beta_{1} d$ 元, 这里 $\beta_{1} \in(0,1)$ 是分红过程中比例费用因子, 而 $1-\beta_{1}$ 为税率. 类似 地, 如果公司需要注资 $\eta$ 元, 其实际注资成本为 $\beta_{2} \eta+K$ 元, 这里 $\beta_{2}>1$ 是注资过程中比例费用因子, $K>0$ 是与注资额度无关的固定费用.

问题 2.1 类似文献 [12], 我们用如下运行函数度量控制策略 $\pi \in \Pi$ 下的公司价值:

$$
V(x, \pi)=\mathrm{E}^{x}\left(\beta_{1} \int_{0}^{T^{\pi}} \mathrm{e}^{-\delta s} d D_{s}^{\pi}+P \mathrm{e}^{-\delta T^{\pi}}-\sum_{i=1}^{\infty} \mathrm{e}^{-\delta \tau_{i}^{\pi}}\left(\beta_{2} \eta_{i}^{\pi}+K\right) I_{\left\{\tau_{i}^{\pi} \leqslant T^{\pi}\right\}}\right),
$$

这里 $\mathrm{E}^{x}$ 表示关于初值 $X_{0-}^{\pi}=x$ 求期望; $\delta>0$ 表示利息力; $I_{\{\cdot\}}$ 是一个示性函数. 事实上, $V(x, \pi)$ 表 示破产前分红与破产终端值期望现值之和减去注资成本的期望现值. 本文从股东利益出发, 在最大化 公司价值目标下寻找最优的策略 $\pi^{*} \in \Pi$ 使得

$$
V(x):=V\left(x, \pi^{*}\right)=\max _{\pi \in \Pi} V(x, \pi),
$$

这里 $V(x)$ 是最大的运行函数, 也称为值函数.

注 2.1 如文献 $[10,21]$ 所述, 溢额再保险是期望值保费准则下最优的再保险形式. 这是因为过 程 (2.5) 在相同的波动率下溢额再保险总是对应更大的漂移项, 因此, 股东有机会在未来获得更多的 红利.

\section{HJB 方程和验证性定理}

利用随机控制的方法, 我们通过求解 HJB 方程给出值函数并构建相应的最优策略. 首先, 对任意 函数 $\omega(x) \in \mathbb{C}^{2}$, 我们定义如下两个算子: 
(1) 注资算子

$$
\mathscr{M} \omega(x)=\max _{y \geqslant 0}\left\{\omega(x+y)-\beta_{2} y-K\right\}
$$

(2) 微分算子

$$
\mathscr{A}^{a} \omega(x)=\frac{1}{2} \lambda \nu(a) \omega^{\prime \prime}(x)+\left[\left(\theta_{1}-\theta_{2}\right) \lambda \mu_{m}+\theta_{2} \lambda \mu(a)\right] \omega^{\prime}(x)-\delta \omega(x) .
$$

假定值函数 $V(x)$ 在区间 $[0, \infty)$ 上二阶连续可导. 利用标准的随机控制理论 (如文献 [25]), 我们 可以给出问题 2.1 对应的 HJB 方程

$$
\begin{aligned}
& \max \left\{\mathscr{M} v(x)-v(x), \max _{0 \leqslant a \leqslant m}\left\{\mathscr{A}^{a} v(x)\right\}, \beta_{1}-v^{\prime}(x)\right\}=0, \\
& \max \{\mathscr{M} v(0)-v(0), P-v(0)\}=0 .
\end{aligned}
$$

引理 3.1 考虑到货币的时间价值, 管理者应当尽量延迟注资, 即注资仅可能在公司濒临破产时 发生. 从而, 对任意 $x>0$ 都有 $\mathscr{M} v(x)-v(x)<0$ 成立. 该结论的类似证明可参见文献 [22, 引理 3.2].

引理 3.2 值函数 $V(x)$ 在区间 $[0, \infty)$ 上递增, 并且对任意 $x \geqslant y \geqslant 0$ 有以下性质存在:

$$
\beta_{1}(x-y) \leqslant V(x)-V(y) \leqslant \beta_{2}(x-y)+K
$$

且

$$
\beta_{1} x+P \leqslant V(x) \leqslant \beta_{1}\left(\frac{\lambda \theta_{1} \mu_{m}}{\delta}+x\right)+P I_{\{P>0\}} .
$$

证明 利用文献 [19, 引理 2.1] 的方法可以类似证明 (3.3), 故此处 (3.3) 的证明省略. 下面将给 出 (3.4) 的证明过程. 根据 (2.10) 中 $\left\{X_{t}^{a}\right\}$ 的表达式, 有

$$
\mathrm{E}^{x}\left(\int_{0}^{T^{\pi}} \mathrm{e}^{-\delta s} d X_{s}^{a}\right)=\mathrm{E}^{x}\left(\int_{0}^{T^{\pi}} \mathrm{e}^{-\delta s}\left[\left(\theta_{1}-\theta_{2}\right) \lambda \mu_{m}+\theta_{2} \lambda \mu(a)\right] d s\right) \leqslant \frac{\lambda \theta_{1} \mu_{m}}{\delta} .
$$

根据 Itô 公式, 我们得到

$$
\mathrm{e}^{-\delta T^{\pi}} X_{T^{\pi}}^{\pi}=x-\delta \int_{0}^{T^{\pi}} \mathrm{e}^{-\delta s} X_{s}^{\pi} d s+\int_{0}^{T^{\pi}} \mathrm{e}^{-\delta s} d X_{s}^{\pi} .
$$

因为对任意 $t \leqslant T^{\pi}$ 都有 $X_{t}^{\pi} \geqslant 0$ 成立且 $X_{T^{\pi}}^{\pi}=0$, 所以, 对上式两边取期望可得

$$
-\mathrm{E}^{x}\left(\int_{0}^{T^{\pi}} \mathrm{e}^{-\delta s} d X_{s}^{\pi}\right)=x-\mathrm{E}^{x}\left(\delta \int_{0}^{T^{\pi}} \mathrm{e}^{-\delta s} X_{s}^{\pi} d s\right) \leqslant x .
$$

从而,

$$
\begin{aligned}
V(x, \pi) & =\mathrm{E}^{x}\left(\beta_{1} \int_{0}^{T^{\pi}} \mathrm{e}^{-\delta s} d D_{s}^{\pi}-\sum_{i=1}^{\infty} \mathrm{e}^{-\delta \tau_{i}^{\pi}}\left(\beta_{2} \eta_{i}^{\pi}+K\right) I_{\left\{\tau_{i}^{\pi} \leqslant T^{\pi}\right\}}+P \mathrm{e}^{-\delta T^{\pi}}\right) \\
& \leqslant \beta_{1} \mathrm{E}^{x}\left(\int_{0}^{T^{\pi}} \mathrm{e}^{-\delta s} d D_{s}^{\pi}-\sum_{i=1}^{\infty} \mathrm{e}^{-\delta \tau_{i}^{\pi}} \eta_{i}^{\pi} I_{\left\{\tau_{i}^{\pi} \leqslant T^{\pi}\right\}}\right)+P I_{\{P>0\}} \\
& =\beta_{1}\left[\mathrm{E}^{x}\left(\int_{0}^{T^{\pi}} \mathrm{e}^{-\delta s} d X_{s}^{a}\right)-\mathrm{E}^{x}\left(\int_{0}^{T^{\pi}} \mathrm{e}^{-\delta s} d X_{s}^{\pi}\right)\right]+P I_{\{P>0\}}
\end{aligned}
$$




$$
\leqslant \beta_{1}\left(x+\frac{\lambda \theta_{1} \mu_{m}}{\delta}\right)+P I_{\{P>0\}}
$$

故引理得证.

定理 3.1 (验证性定理) 假如存在某二阶连续可导、递增的凹函数 $v(x)$ 满足 HJB 方程 $(3.1)$ 和 $(3.2)$, 并且导函数 $v^{\prime}(x)$ 有界, 则

(1) 对任意策略 $\pi \in \Pi$ 都有 $v(x) \geqslant V(x, \pi)$ 成立, 故 $v(x) \geqslant V(x)$;

(2) 更进一步, 如果存在策略 $\pi^{*}=\left(a^{\pi^{*}} ; D^{\pi^{*}} ; R^{\pi^{*}}\right) \in \Pi$ 满足 $v(x)=V\left(x, \pi^{*}\right)$, 那么 $v(x)=V(x)$ 并 且 $\pi^{*}$ 是最优的策略.

定理 3.1 的具体证明过程见附录 A.

定理 3.1 为求解问题 2.1 提供了思路. 我们将求解 HJB 给出值函数, 并构建相应的最优策略 $\pi^{*} . \theta_{2}$ 反映了再保险成本, 它的大小会影响到保险公司的再保险策略. 当 $\theta_{2}$ 较小即再保险成本并不太大时 保险公司可能会选择购买再保险. 反之, 保险公司会放弃购买再保险. 接下来, 我们根据 $\theta_{2}$ 与临界值 $\frac{m}{\lambda \nu_{m}}\left(\lambda \theta_{1} \mu_{m}+\sqrt{\left(\lambda \theta_{1} \mu_{m}\right)^{2}+2 \lambda \delta \nu_{m}}\right)$ 之间的大小关系分别讨论最优化问题的解. 根据 (2.8) 可知,

$$
\frac{m}{\lambda \nu_{m}}\left(\lambda \theta_{1} \mu_{m}+\sqrt{\left(\lambda \theta_{1} \mu_{m}\right)^{2}+2 \lambda \delta \nu_{m}}\right)>2 \theta_{1} .
$$

\section{4 第一种情形: $\theta_{2} \in\left(\theta_{1}, \frac{m}{\lambda \nu_{m}}\left(\lambda \theta_{1} \mu_{m}+\sqrt{\left(\lambda \theta_{1} \mu_{m}\right)^{2}+2 \lambda \delta \nu_{m}}\right)\right)$}

本节考虑安全负荷满足如下条件:

$$
\theta_{1}<\theta_{2}<\frac{m}{\lambda \nu_{m}}\left(\lambda \theta_{1} \mu_{m}+\sqrt{\left(\lambda \theta_{1} \mu_{m}\right)^{2}+2 \lambda \delta \nu_{m}}\right) .
$$

此时可以理解为再保险人安全负荷适中, 并未索取高价, 原保险人可以考虑购买再保险. 考虑到 (3.2) 的结构特点, 我们将在不同的边界条件下求解 HJB 方程.

\section{1 无注资情形}

假设当保险公司盈余为 0 时最优的选择是立即宣布退出市场, 即注资无利可图. 根据最优控制理 论, 相应的边界条件是 $v(0)=P$ 和 $\mathscr{M} v(0)-v(0) \leqslant 0$. 假设 $f(x)$ 是 HJB 方程二次连续可导且递增的 凹解, 那么, 它应该满足如下方程组:

$$
\begin{aligned}
& \max _{0 \leqslant a \leqslant m}\left\{\mathscr{A}^{a} f(x)\right\}=0, \quad 0 \leqslant x \leqslant b_{0}, \\
& \beta_{1}-f^{\prime}(x)=0, \quad x \geqslant b_{0}, \\
& f(0)=P, \\
& \mathscr{M} f(0)-f(0) \leqslant 0,
\end{aligned}
$$

这里分红临界点 $b_{0} \geqslant 0$ 待定. 接下来将讨论 (4.2)-(4.5) 的解并构造相应的最优策略.

首先对 (4.2) 关于变量 $a$ 求导并令其导函数为 0 得到

$$
a(x)=-\theta_{2} \frac{f^{\prime}(x)}{f^{\prime \prime}(x)} .
$$


将 (4.6) 代入 (4.2) 得到

$$
\lambda\left[\theta_{2} h(a(x))+\left(\theta_{1}-\theta_{2}\right) \mu_{m}\right] f^{\prime}(x)-\delta f(x)=0,
$$

这里

$$
h(a):=\mu(a)-\frac{\nu(a)}{2 a} \in \mathbb{C}^{1}(0, \infty) .
$$

利用 (2.8), 我们计算可知 $h(0+)=0, h(m)=\mu_{m}-\frac{\nu_{m}}{2 m}>0$, 且易证 $h^{\prime}(a)=\frac{\nu(a)}{2 a^{2}}>0$ 对任意 $a \in[0, m]$ 成立. 对 (4.7) 两边关于变量 $x$ 求导并再次利用 (4.6) 得到

$$
a^{\prime}(x)=\frac{\delta a(x)+\lambda \theta_{2}^{2} h(a(x))+\lambda \theta_{2}\left(\theta_{1}-\theta_{2}\right) \mu_{m}}{\lambda \theta_{2} a(x) h^{\prime}(a(x))} .
$$

为方便起见, 定义

$$
\chi(x):=\delta x+\lambda \theta_{2}^{2} h(x)+\lambda \theta_{2}\left(\theta_{1}-\theta_{2}\right) \mu_{m} .
$$

由于对任意 $x \geqslant 0$ 有 $\chi^{\prime}(x)=\delta+\lambda \theta_{2}^{2} h^{\prime}(x)>0$ 成立, 故函数 $\chi(x)$ 是严格递增的. 另外, 在 (4.1) 前提 假设下, 易验证如下性质:

$$
\begin{aligned}
& \chi(0)=\lambda \theta_{2}\left(\theta_{1}-\theta_{2}\right) \mu_{m}<0, \\
& \chi(m)=-\frac{\lambda \nu_{m}}{2 m} \theta_{2}^{2}+\lambda \theta_{1} \theta_{2} \mu_{m}+m \delta>0 .
\end{aligned}
$$

因此, 在区间 $(0, m)$ 上存在唯一的解 $\alpha$ 满足方程 $\chi(\alpha)=0$. 显然, 对任意 $x \in(\alpha, \infty)$ 总有不等式 $\chi(x)>0$ 成立. 假设 $a(0):=a_{0} \in(\alpha, m]$, 定义如下函数:

$$
Q(x)=\int_{a_{0}}^{x} \frac{\lambda \theta_{2} y h^{\prime}(y)}{\delta y+\lambda \theta_{2}^{2} h(y)+\lambda \theta_{2}\left(\theta_{1}-\theta_{2}\right) \mu_{m}} d y, \quad a_{0} \leqslant x \leqslant m .
$$

从 $\chi(x)>0$ 和 $h^{\prime}(x)>0$ 可知上式中的被积函数是正数, 所以, $Q(x)$ 严格递增且满足 $Q(m)<\infty$. 从 而, 在区间 $\left[0, x_{0}\right]$ 上 $Q(x)$ 的反函数 $Q^{-1}(x)$ 存在, 这里 $x_{0}=Q(m)$. 另外, 我们猜想再保险策略临界 点 $x_{0}$ 小于分红策略临界点 $b_{0}$, 该猜想将在后面得到验证. 考虑到 $Q\left(a_{0}\right)=0$, 并利用反函数的导数性 质可知,

$$
a(x)=Q^{-1}(x), \quad 0 \leqslant x \leqslant x_{0} .
$$

综合 (4.4)、(4.6) 和 (4.12), 我们给出如下表达式:

$$
f(x)=k_{3} \int_{0}^{x} \mathrm{e}^{\int_{y}^{x_{0}} \frac{\theta_{2}}{Q^{-1}(z)} d z} d y+P, \quad 0 \leqslant x \leqslant x_{0},
$$

这里参数 $k_{3}>0$ 待定.

考虑到风险自留水平的连续性和递增性, 我们猜测当盈余超过 $x_{0}$ 时保险公司应当自留全部风险, 即对任意 $x \in\left[x_{0}, b_{0}\right]$ 都有 $a(x) \equiv m$. 那么, (4.2) 简化为一个二阶常微分方程

$$
\frac{1}{2} \lambda \nu_{m} f^{\prime \prime}(x)+\theta_{1} \lambda \mu_{m} f^{\prime}(x)-\delta f(x)=0,
$$

解得

$$
f(x)=k_{1} \mathrm{e}^{r_{+}\left(x-x_{0}\right)}+k_{2} \mathrm{e}^{r_{-}\left(x-x_{0}\right)}, \quad x_{0} \leqslant x \leqslant b_{0},
$$


这里 $k_{1}$ 和 $k_{2}$ 是待定参数, 且

$$
\begin{aligned}
& r_{+}=\frac{1}{\lambda \nu_{m}}\left(-\lambda \theta_{1} \mu_{m}+\sqrt{\left(\lambda \theta_{1} \mu_{m}\right)^{2}+2 \lambda \delta \nu_{m}}\right)>0, \\
& r_{-}=\frac{1}{\lambda \nu_{m}}\left(-\lambda \theta_{1} \mu_{m}-\sqrt{\left(\lambda \theta_{1} \mu_{m}\right)^{2}+2 \lambda \delta \nu_{m}}\right)<0 .
\end{aligned}
$$

最后, 根据方程 $(4.3)$ 和函数 $f(x)$ 的光滑性可得

$$
f(x)=\beta_{1}\left(x-b_{0}\right)+f\left(b_{0}\right), \quad x \geqslant b_{0} .
$$

利用导函数 $f^{\prime}(x)$ 和 $f^{\prime \prime}(x)$ 在 $x_{0}$ 和 $b_{0}$ 点处的连续性, 我们得到

$$
\begin{aligned}
& k_{1} r_{+}+k_{2} r_{-}=k_{3}, \\
& k_{1}\left(r_{+}\right)^{2}+k_{2}\left(r_{-}\right)^{2}=-\frac{\theta_{2}}{m} k_{3}, \\
& k_{1} r_{+} \mathrm{e}^{r_{+}\left(b_{0}-x_{0}\right)}+k_{2} r_{-} \mathrm{e}^{r_{-}\left(b_{0}-x_{0}\right)}=\beta_{1}, \\
& k_{1}\left(r_{+}\right)^{2} \mathrm{e}^{r_{+}\left(b_{0}-x_{0}\right)}+k_{2}\left(r_{-}\right)^{2} \mathrm{e}^{r_{-}\left(b_{0}-x_{0}\right)}=0 .
\end{aligned}
$$

解方程 (4.19) 和 (4.20) 得

$$
k_{1}=k_{3} c_{1}, \quad k_{2}=k_{3} c_{2}
$$

这里

$$
\begin{gathered}
c_{1}=\frac{r_{-}+\frac{\theta_{2}}{m}}{r_{+}\left(r_{-}-r_{+}\right)}>0, \\
c_{2}=\frac{r_{+}+\frac{\theta_{2}}{m}}{r_{-}\left(r_{+}-r_{-}\right)}<0,
\end{gathered}
$$

(4.24) 中不等式在 (4.1) 前提条件下必然成立. 将 (4.23) 代入 (4.22) 得

$$
b_{0}=x_{0}+\frac{1}{r_{+}-r_{-}} \ln \left(\frac{m+\frac{\theta_{2}}{r_{+}}}{m+\frac{\theta_{2}}{r_{-}}}\right)>x_{0},
$$

因在 (4.1) 的前提条件下有 $m+\frac{\theta_{2}}{r_{+}}>m+\frac{\theta_{2}}{r_{-}}>0$ 成立, 故 (4.26) 中的不等式成立. 利用 (4.23) 和 (4.26), 解 (4.21) 得到

$$
k_{3}=\frac{\beta_{1} m}{m+\frac{\theta_{2}}{r_{+}}}\left(\frac{m+\frac{\theta_{2}}{r_{+}}}{m+\frac{\theta_{2}}{r_{-}}}\right)^{\frac{-r_{-}}{r_{+}-r_{-}}}>0 .
$$

引理 $4.1 \beta_{1}<k_{3}<\frac{\beta_{1} m}{m+\frac{\theta_{2}}{r_{-}}}$和 $\lim _{\theta_{2} \rightarrow-m r_{-}} k_{3}=\infty$.

证明 一方面, 我们对 (4.27) 中的 $k_{3}$ 取对数并借助对数函数的凹性可知,

$$
\begin{aligned}
\log k_{3} & =\log \beta_{1}+\log m-\log \left(m+\frac{\theta_{2}}{r_{+}}\right)-\frac{r_{-}}{r_{+}-r_{-}}\left[\log \left(m+\frac{\theta_{2}}{r_{+}}\right)-\log \left(m+\frac{\theta_{2}}{r_{-}}\right)\right] \\
& =\log \beta_{1}+\log m-\left[\frac{r_{+}}{r_{+}-r_{-}} \log \left(m+\frac{\theta_{2}}{r_{+}}\right)-\frac{r_{-}}{r_{+}-r_{-}} \log \left(m+\frac{\theta_{2}}{r_{-}}\right)\right]
\end{aligned}
$$

976 


$$
\begin{aligned}
& >\log \beta_{1}+\log m-\log \left[\frac{r_{+}}{r_{+}-r_{-}}\left(m+\frac{\theta_{2}}{r_{+}}\right)-\frac{r_{-}}{r_{+}-r_{-}}\left(m+\frac{\theta_{2}}{r_{-}}\right)\right] \\
& =\log \beta_{1}+\log m-\log m=\log \beta_{1},
\end{aligned}
$$

因此, $\beta_{1}<k_{3}$ 成立. 另一方面, 利用不等式

$$
m+\frac{\theta_{2}}{r_{+}}>m+\frac{\theta_{2}}{r_{-}}>0 \quad \text { 和 } \quad 0<\frac{-r_{-}}{r_{+}-r_{-}}<1,
$$

可得

$$
k_{3}=\frac{\beta_{1} m}{m+\frac{\theta_{2}}{r_{+}}}\left(\frac{m+\frac{\theta_{2}}{r_{+}}}{m+\frac{\theta_{2}}{r_{-}}}\right)^{\frac{-r_{-}}{r_{+}-r_{-}}}<\frac{\beta_{1} m}{m+\frac{\theta_{2}}{r_{+}}} \cdot \frac{m+\frac{\theta_{2}}{r_{+}}}{m+\frac{\theta_{2}}{r_{-}}}=\frac{\beta_{1} m}{m+\frac{\theta_{2}}{r_{-}}} .
$$

从而不等式得证. 有趣的是, (4.1) 可以写成 $\theta_{1}<\theta_{2}<-m r_{-}$. 另外, 容易证明

$$
\frac{d}{d \theta_{2}} \log k_{3}=\frac{\lambda \theta_{2} \nu_{m}}{2 \delta\left(m+\frac{\theta_{2}}{r_{+}}\right)\left(m+\frac{\theta_{2}}{r_{-}}\right)}>0 .
$$

因此, $k_{3}:=k_{3}\left(\theta_{2}\right)$ 在区间 $\left(\theta_{1},-m r_{-}\right)$上递增且 $\lim _{\theta_{2} \rightarrow-m r_{-}} k_{3}=\infty$.

至此, 我们得到了方程组 (4.2)-(4.4) 的二阶连续可导且递增的凹函数解:

$$
f(x)= \begin{cases}k_{3} \int_{0}^{x} \mathrm{e}^{\int_{y}^{x_{0}} \frac{\theta_{2}}{Q^{-1}(z)} d z} d y+P, & 0 \leqslant x \leqslant x_{0}, \\ k_{1} \mathrm{e}^{r_{+}\left(x-x_{0}\right)}+k_{2} \mathrm{e}^{r_{-}\left(x-x_{0}\right)}, & x_{0} \leqslant x \leqslant b_{0}, \\ \beta_{1}\left(x-b_{0}\right)+f\left(b_{0}\right), & x \geqslant b_{0},\end{cases}
$$

相应的最优自留风险水平是

$$
a^{\pi^{*}}(x)= \begin{cases}Q^{-1}(x), & 0 \leqslant x \leqslant x_{0} \\ m, & x \geqslant x_{0}\end{cases}
$$

(4.23)-(4.25) 和 (4.27) 已经给出了 $k_{1} 、 k_{2}$ 和 $k_{3}$ 的表达式, 接下来将确定参数 $a_{0} 、 x_{0}$ 和 $b_{0}$. 令 (4.7) 中 $x=0$ 得到

$$
\lambda k_{3}\left[\theta_{2} h\left(a_{0}\right)+\left(\theta_{1}-\theta_{2}\right) \mu_{m}\right] \mathrm{e}^{\int_{0}^{x_{0}} \frac{\theta_{2}}{Q^{-1}(z)} d z}=\delta P .
$$

作变量代换 $y=Q^{-1}(z)$ 并结合 $(4.8)$ 可得

$$
P=\frac{\lambda k_{3}}{\delta} \Phi\left(a_{0}\right), \quad \alpha<a_{0} \leqslant m,
$$

这里方程 $\Phi(x)$ 具有如下表达形式:

$$
\Phi(x)=\left[\theta_{2} h(x)+\left(\theta_{1}-\theta_{2}\right) \mu_{m}\right] \exp \left\{\int_{x}^{m} \frac{\lambda \theta_{2}^{2} h^{\prime}(y)}{\delta y+\lambda \theta_{2}^{2} h(y)+\lambda \theta_{2}\left(\theta_{1}-\theta_{2}\right) \mu_{m}} d y\right\} .
$$

利用函数 $\chi(x)$ 的性质可知,

$$
\Phi^{\prime}(x)=\theta_{2} h^{\prime}(x) \exp \left\{\int_{x}^{m} \frac{\lambda \theta_{2}^{2} h^{\prime}(y)}{\delta y+\lambda \theta_{2}^{2} h(y)+\lambda \theta_{2}\left(\theta_{1}-\theta_{2}\right) \mu_{m}} d y\right\}
$$




$$
\begin{aligned}
& \times \frac{\delta x}{\delta x+\lambda \theta_{2}^{2} h(x)+\lambda \theta_{2}\left(\theta_{1}-\theta_{2}\right) \mu_{m}} \\
& >0 .
\end{aligned}
$$

所以, 函数 $\Phi(x)$ 在区间 $[\alpha, m]$ 上递增且最大值为 $\Phi(m)=\theta_{2} h(m)+\left(\theta_{1}-\theta_{2}\right) \mu_{m}$. 不难证明,

$$
\frac{\lambda \theta_{2}^{2} h^{\prime}(y)}{\delta y+\lambda \theta_{2}^{2} h(y)+\lambda \theta_{2}\left(\theta_{1}-\theta_{2}\right) \mu_{m}} \sim \frac{\gamma}{y-\alpha},
$$

这里 $\gamma:=\frac{\delta+\lambda \theta_{2}^{2} h^{\prime}(\alpha)}{\lambda \theta_{2}^{2} h^{\prime}(\alpha)}>0$, “ ” 表示 $y \rightarrow \alpha+$ 时等价无穷大. 注意到

$$
\theta_{2} h(\alpha)+\left(\theta_{1}-\theta_{2}\right) \mu_{m}=\frac{\chi(\alpha)-\delta \alpha}{\lambda \theta_{2}}=\frac{-\delta \alpha}{\lambda \theta_{2}}<0, \quad \lim _{x \rightarrow \alpha+} \int_{x}^{m} \frac{\gamma}{y-\alpha} d y=+\infty,
$$

因此有 $\lim _{x \rightarrow \alpha+} \Phi(x)=-\infty$. 根据以上分析, 我们可断定当且仅当

$$
P<\frac{\lambda k_{3}}{\delta}\left[\theta_{2} h(m)+\left(\theta_{1}-\theta_{2}\right) \mu_{m}\right]
$$

时方程 (4.33) 存在唯一的根 $a_{0}=a(0) \in(\alpha, m)$. 进而 (4.26) 中参数 $b_{0}$ 也可以得到.

接下来需要验证 (4.30) 中给出的 $f(x)$ 满足 (4.5), 我们分 3 种情形讨论.

(i) 当 $\beta_{1}<f^{\prime}(0)=k_{3} \mathrm{e}^{\int_{0}^{x_{0}} \frac{\theta_{2}}{Q^{-1}(z)} d z} \leqslant \beta_{2}$ 时, 由于 $f^{\prime}(x)$ 在区间 $[0, \infty)$ 上递减, 故对任意 $x \geqslant 0$ 有 $f^{\prime}(x) \leqslant \beta_{2}$ 成立. 因此,

$$
\mathscr{M} f(0)-f(0)=\max _{y \geqslant 0}\left\{f(y)-\beta_{2} y-K\right\}-f(0)=-K<0,
$$

于是 $(4.5)$ 得证.

(ii) 当 $f^{\prime}(0)=k_{3} \mathrm{e}^{\int_{0}^{x_{0}}} \frac{\theta_{2}}{Q^{-1}(z)} d z>\beta_{2} \geqslant k_{3}$ 时, 由于函数 $f^{\prime}(x)$ 在区间 $\left[0, b_{0}\right]$ 上递减且值域为 $\left[\beta_{1}, f^{\prime}(0)\right]$, 所以, 存在唯一的数 $\xi_{1}^{*} \in\left(0, x_{0}\right)$ 使得方程 $f^{\prime}\left(\xi_{1}^{*}\right)=\beta_{2}$ 成立. 定义积分

$$
I_{1}\left(\xi_{1}^{*}\right):=\int_{0}^{\xi_{1}^{*}}\left(f^{\prime}(x)-\beta_{2}\right) d x=f\left(\xi_{1}^{*}\right)-f(0)-\beta_{2} \xi_{1},
$$

这里函数 $f(x)$ 如 (4.30) 所示. 显然, 当且仅当满足

$$
K \geqslant I_{1}\left(\xi_{1}^{*}\right)
$$

条件时有 (4.5) 成立.

(iii) 当 $f^{\prime}(0)=k_{3} \mathrm{e}^{\int_{0}^{x_{0}} \frac{\theta_{2}}{Q^{-1}(z)} d z}>k_{3}>\beta_{2}$ 时, 由于函数 $f^{\prime}(x)$ 在区间 $\left[0, b_{0}\right]$ 上递减且值域为 $\left[f^{\prime}(0), \beta_{1}\right]$, 故存在唯一的解 $\xi_{2}^{*} \in\left(x_{0}, b_{0}\right)$ 满足方程 $f^{\prime}\left(\xi_{2}^{*}\right)=\beta_{2}$. 定义积分

$$
\begin{aligned}
& I_{2}\left(\xi_{2}^{*}\right):=\int_{0}^{\xi_{2}^{*}}\left(f^{\prime}(x)-\beta_{2}\right) d x=f\left(\xi_{2}^{*}\right)-f(0)-\beta_{2} \xi_{2}^{*}, \\
& J\left(\xi_{2}^{*}\right):=\int_{x_{0}}^{\xi_{2}^{*}}\left(f^{\prime}(x)-\beta_{2}\right) d x=f\left(\xi_{2}^{*}\right)-f\left(x_{0}\right)-\beta_{2}\left(\xi_{2}^{*}-x_{0}\right),
\end{aligned}
$$

这里 $f(x)$ 如 (4.30) 所示. 显然, 当且仅当

$$
K \geqslant I_{2}\left(\xi_{2}^{*}\right)
$$


时有 (4.5) 成立. 后面章节中将用到 (4.39).

当 $P \geqslant \frac{\lambda k_{3}}{\delta}\left[\theta_{2} h(m)+\left(\theta_{1}-\theta_{2}\right) \mu_{m}\right]$ 时, 方程 (4.33) 在区间 $(\alpha, m)$ 上无解. 换句话讲, 对任意 $x \geqslant 0$ 均不可能出现 $a(x)<m$. 因此,

$$
a^{\pi^{*}}(x) \equiv m, \quad x \geqslant 0,
$$

即保险公司始终不购买再保险. 相应地, (4.2)-(4.4) 的解是

$$
f(x)= \begin{cases}s_{1} \mathrm{e}^{r_{+} x}+s_{2} \mathrm{e}^{r_{-} x}, & 0 \leqslant x \leqslant b_{0}, \\ \beta_{1}\left(x-b_{0}\right)+f\left(b_{0}\right), & x \geqslant b_{0},\end{cases}
$$

这里参数 $s_{1}$ 和 $s_{2}$ 待定. 利用函数 $f(x)$ 在 $b_{0}$ 点处的光滑性可知,

$$
\begin{aligned}
& s_{1}=\frac{\beta_{1} r_{-}}{r_{+}\left(r_{-}-r_{+}\right)} \mathrm{e}^{-r_{+} b_{0}}, \\
& s_{2}=\frac{\beta_{1} r_{+}}{r_{-}\left(r_{+}-r_{-}\right)} \mathrm{e}^{-r_{-} b_{0}} .
\end{aligned}
$$

边界条件 $f(0)=s_{1}+s_{2}=P$ 可以得到 $b_{0}$ 满足的方程

$$
\frac{\beta_{1} r_{-}}{r_{+}\left(r_{-}-r_{+}\right)} \mathrm{e}^{-r_{+} b_{0}}+\frac{\beta_{1} r_{+}}{r_{-}\left(r_{+}-r_{-}\right)} \mathrm{e}^{-r_{-} b_{0}}=P .
$$

为了表述方便, 我们定义如下函数:

$$
w(x):=\frac{\beta_{1} r_{-}}{r_{+}\left(r_{-}-r_{+}\right)} \mathrm{e}^{-r_{+} x}+\frac{\beta_{1} r_{+}}{r_{-}\left(r_{+}-r_{-}\right)} \mathrm{e}^{-r_{-} x}, \quad x \geqslant 0 .
$$

易证 $w(0)=\frac{\beta_{1}\left(r_{+}+r_{-}\right)}{r_{+} r_{-}}=\beta_{1} \lambda \theta_{1} \mu_{m} / \delta>0, w^{\prime}(x)<0$ 和 $w(\infty)=-\infty$, 因此, 函数 $w(x)$ 是递减的, 并且 值域为 $\left[-\infty, \beta_{1} \lambda \theta_{1} \mu_{m} / \delta\right]$. 因此, 在

$$
\frac{\lambda k_{3}}{\delta}\left[\theta_{2} h(m)+\left(\theta_{1}-\theta_{2}\right) \mu_{m}\right] \leqslant P<\frac{\beta_{1} \lambda \theta_{1} \mu_{m}}{\delta}
$$

情形下, 方程 (4.45) 有唯一的解 $b_{0}>0$. 值得注意的是, (4.46) 要求

$$
\frac{\lambda k_{3}}{\delta}\left[\theta_{2} h(m)+\left(\theta_{1}-\theta_{2}\right) \mu_{m}\right]<\frac{\beta_{1} \lambda \theta_{1} \mu_{m}}{\delta} .
$$

将 $k_{3}$ 和 $h(a)$ 的表达式代入该不等式得

$$
\frac{m}{m+\frac{\theta_{2}}{r_{+}}}\left(\frac{m+\frac{\theta_{2}}{r_{+}}}{m+\frac{\theta_{2}}{r_{-}}}\right)^{\frac{-r_{-}}{r_{+}-r_{-}}}\left(\mu_{m} \theta_{1}-\frac{\nu_{m}}{2 m} \theta_{2}\right)<\mu_{m} \theta_{1},
$$

其证明可见附录 B. 最后, 我们需要验证 (4.41) 中的 $a^{\pi^{*}}$ 和 (4.42) 中的 $f(x)$ 满足 (4.5).

(i) 当 $f^{\prime}(0)=s_{1} r_{+}+s_{2} r_{-}=\frac{\beta_{1} r_{-}}{r_{-}-r_{+}} \mathrm{e}^{-r_{+} b_{1}}+\frac{\beta_{1} r_{+}}{r_{+}-r_{-}} \mathrm{e}^{-r_{-} b_{1}} \leqslant \beta_{2}$ 时, 因为 $f^{\prime}(x)$ 在区间 $[0, \infty)$ 上递 减, 故 $f^{\prime}(x) \leqslant \beta_{2}$ 成立. 因此,

$$
\mathscr{M} f(0)-f(0)=\max _{y \geqslant 0}\left\{f(y)-\beta_{2} y-K\right\}-f(0)=-K<0,
$$

(4.5) 得证. 
(ii) 当 $f^{\prime}(0)=s_{1} r_{+}+s_{2} r_{-}=\frac{\beta_{1} r_{-}}{r_{-}-r_{+}} \mathrm{e}^{-r_{+} b_{1}}+\frac{\beta_{1} r_{+}}{r_{+}-r_{-}} \mathrm{e}^{-r_{-} b_{1}}>\beta_{2}$ 时, 因为导函数 $f^{\prime}(x)$ 递减, 且值 域为 $\left[\beta_{1}, f^{\prime}(0)\right]$, 所以, 存在唯一的根 $\xi_{3}^{*} \in\left(0, b_{0}\right)$ 满足方程 $f^{\prime}\left(\xi_{3}^{*}\right)=\beta_{2}$. 定义积分

$$
I_{3}\left(\xi_{3}^{*}\right):=\int_{0}^{\xi_{3}^{*}}\left(f^{\prime}(x)-\beta_{2}\right) d x=f\left(\xi_{3}^{*}\right)-f(0)-\beta_{2} \xi_{3}^{*},
$$

这里 $f(x)$ 如 (4.42) 所示. 显然, (4.5) 成立当且仅当

$$
K \geqslant I_{3}\left(\xi_{3}^{*}\right)
$$

最后, 在 $P \geqslant \beta_{1} \lambda \theta_{1} \mu_{m} / \delta$ 情形下, 方程 (4.45) 不存在正解. 此时, 我们猜想 $b_{0}=0$, 这意味着保险 公司应立即将当前所有盈余作为红利分发给股东, 并索取终端值 $P$. 相应地, (4.2)-(4.5) 的解为

$$
f(x)=\beta_{1} x+P, \quad x \geqslant 0,
$$

对应的最优自留水平 $a^{\pi^{*}}$ 同 (4.41).

引理 4.2 以上分析过程中, 我们得到了方程组 (4.2)-(4.5) 的解 $f(x)$ 和相应的最优风险自留水 平 $a^{\pi^{*}}(x)$, 且 $f(x)$ 是二阶连续可导且递增的凹函数, 其一阶导函数有界. 事实上, 它们也满足 HJB 方 程 (3.1) 和 (3.2). 主要结果总结如下:

(1) 如果 $P<\frac{\lambda k_{3}}{\delta}\left[\theta_{2} h(m)+\left(\theta_{1}-\theta_{2}\right) \mu_{m}\right]$ 且 $\beta_{1}<f^{\prime}(0)=k_{3} \mathrm{e}^{\int_{0}^{x_{0}} \frac{\theta_{2}}{Q^{-1}(z)} d z} \leqslant \beta_{2}$ 成立, 则解 $f(x)$ 和 $a^{\pi^{*}}(x)$ 分别由 (4.30) 和 (4.31) 给出;

(2) 如果 $P<\frac{\lambda k_{3}}{\delta}\left[\theta_{2} h(m)+\left(\theta_{1}-\theta_{2}\right) \mu_{m}\right], f^{\prime}(0)=k_{3} \mathrm{e}^{\int_{0}^{x_{0}} \frac{\theta_{2}}{Q^{-1}(z)} d z}>\beta_{2} \geqslant k_{3}$ 且 $K \geqslant I_{1}\left(\xi_{1}^{*}\right)$ 成立, 则 解 $f(x)$ 和 $a^{\pi^{*}}(x)$ 也可分别由 (4.30) 和 (4.31) 给出;

(3) 如果 $P<\frac{\lambda k_{3}}{\delta}\left[\theta_{2} h(m)+\left(\theta_{1}-\theta_{2}\right) \mu_{m}\right], f^{\prime}(0)=k_{3} \mathrm{e}^{\int_{0}^{x_{0}} \frac{\theta_{2}}{Q^{-1}(z)} d z}>k_{3}>\beta_{2}$ 且 $K \geqslant I_{2}\left(\xi_{2}^{*}\right)$ 成立, 则 解 $f(x)$ 和 $a^{\pi^{*}}(x)$ 也可分别由 (4.30) 和 (4.31) 给出;

(4) 如果 $\frac{\lambda k_{3}}{\delta}\left[\theta_{2} h(m)+\left(\theta_{1}-\theta_{2}\right) \mu_{m}\right] \leqslant P<\beta_{1} \lambda \theta_{1} \mu_{m} / \delta$ 且 $f^{\prime}(0)=\frac{\beta_{1} r_{-}}{r_{-}-r_{+}} \mathrm{e}^{-r_{+} b_{1}}+\frac{\beta_{1} r_{+}}{r_{+}-r_{-}} \mathrm{e}^{-r_{-} b_{1}} \leqslant \beta_{2}$ 成立, 则解 $f(x)$ 和 $a^{\pi^{*}}(x)$ 分别由 (4.42) 和 (4.41) 给出;

(5) 如果 $\frac{\lambda k_{3}}{\delta}\left[\theta_{2} h(m)+\left(\theta_{1}-\theta_{2}\right) \mu_{m}\right] \leqslant P<\beta_{1} \lambda \theta_{1} \mu_{m} / \delta, f^{\prime}(0)=\frac{\beta_{1} r_{-}}{r_{-}-r_{+}} \mathrm{e}^{-r_{+} b_{1}}+\frac{\beta_{1} r_{+}}{r_{+}-r_{-}} \mathrm{e}^{-r_{-} b_{1}}>\beta_{2}$ 且 $K \geqslant I_{3}\left(\xi_{3}^{*}\right)$ 成立, 则解 $f(x)$ 和 $a^{\pi^{*}}(x)$ 分别由 (4.42) 和 (4.41) 给出;

(6) 如果 $P \geqslant \beta_{1} \lambda \theta_{1} \mu_{m} / \delta$, 则解 $f(x)$ 和 $a^{\pi^{*}}(x)$ 分别由 (4.50) 和 (4.41) 给出.

证明 容易证明, 以上 6 种情形下给出的 $f(x)$ 都是二阶连续可导且递增的凹函数. 在本节分析 中, 我们已经证明了以上 $f(x)$ 和 $a^{\pi^{*}}(x)$ 满足 $(4.2)-(4.5)$ 并且不等式 $\beta_{1} \leqslant f^{\prime}(x) \leqslant f^{\prime}(0+)<\infty$ 成立. 我们还需要证明 $f(x)$ 和 $a^{\pi^{*}}(x)$ 满足 HJB 方程 (3.1) 和 (3.2). 不失一般性, 我们在附录 C 给出了 (2) 的证明, 其他结论均可类似证明.

\section{2 有注资情形}

以上分析表明在 (4.37)、(4.40) 或 (4.49) 不成立时, 方程组 (4.2)-(4.5) 没有恰当的解. 这表明保 险公司在盈余为 0 时宣告破产并非最优, 而是应当选择注资. 那么, 相应的边界条件变为 $v(0) \geqslant P$ 和 $\mathscr{M} v(0)-v(0)=0$, HJB 方程中 $v(x)$ 的解 $\tilde{f}(x)$ 应该满足

$$
\begin{aligned}
& \max _{0 \leqslant \tilde{a} \leqslant m}\left\{\mathscr{A}^{\tilde{a}} \tilde{f}(x)\right\}=0, \quad 0 \leqslant x \leqslant \tilde{b}_{0}, \\
& \beta_{1}-\tilde{f}^{\prime}(x)=0, \quad x \geqslant \tilde{b}_{0},
\end{aligned}
$$




$$
\begin{aligned}
& \tilde{f}(0) \geqslant P, \\
& \mathscr{M} \tilde{f}(0)-\tilde{f}(0)=0,
\end{aligned}
$$

这里参数 $\tilde{b}_{0} \geqslant 0$ 待定. 本节将在 (4.37)、(4.40) 和 (4.49) 不成立时求解 (4.51)-(4.54), 并分 4 种情形 讨论:

(i) 当 $P<\frac{\lambda k_{3}}{\delta}\left[\theta_{2} h(m)+\left(\theta_{1}-\theta_{2}\right) \mu_{m}\right], f^{\prime}(0)=k_{3} \mathrm{e}^{\int_{0}^{x_{0}} \frac{\theta_{2}}{Q^{-1}(z)} d z}>\beta_{2} \geqslant k_{3}$ 和 $K<I_{1}\left(\xi_{1}^{*}\right)$ 时, 受文 献 [9] 启发, 我们构建一个可能的解

$$
\tilde{f}_{1}(x)=f\left(x+p_{1}^{*}\right)= \begin{cases}k_{3} \int_{0}^{x+p_{1}^{*}} \mathrm{e}^{\int_{y}^{x_{0}} \frac{\theta_{2}}{Q^{-1}(z)} d z} d y+P, & 0 \leqslant x<x_{0}-p_{1}^{*}, \\ k_{1} \mathrm{e}^{r_{+}\left(x+p_{1}^{*}-x_{0}\right)}+k_{2} \mathrm{e}^{r_{-}\left(x+p_{1}^{*}-x_{0}\right)}, & x_{0}-p_{1}^{*} \leqslant x \leqslant b_{1}, \\ \beta_{1}\left(x-b_{1}\right)+\tilde{f}_{1}\left(b_{1}\right), & x \geqslant b_{1},\end{cases}
$$

这里 $f(x)$ 同 (4.30), $b_{1}=b_{0}-p_{1}^{*}, b_{0}$ 由 (4.26) 给定, 参数 $p_{1}^{*}$ 待求. 考虑到 (4.6), 相应的最优自留水平 应该是

$$
\tilde{a}_{1}^{\pi^{*}}(x)=a^{\pi^{*}}\left(x+p_{1}^{*}\right)= \begin{cases}Q^{-1}\left(x+p_{1}^{*}\right), & 0 \leqslant x \leqslant x_{0}-p_{1}^{*}, \\ m, & x \geqslant x_{0}-p_{1}^{*},\end{cases}
$$

这里 $a^{\pi^{*}}(x)$ 由 (4.31) 给定. 显然, $\tilde{f}_{1}(x)$ 和 $\tilde{a}_{1}^{\pi^{*}}(x)$ 可以由 $f(x)$ 和 $a^{\pi^{*}}(x)$ 向左平移 $p_{1}^{*}$ 个单位得到, 因 此, 它们满足 (4.51)-(4.53). 接下来需要确定 $p_{1}^{*}>0$ 的值使得 (4.54) 成立. 定义函数

$$
\varphi(p):=f\left(\xi_{1}^{*}\right)-f(p)-\beta_{2}\left(\xi_{1}^{*}-p\right)-K, \quad 0 \leqslant p \leqslant \xi_{1}^{*},
$$

这里 $f(x)$ 同 (4.30). 条件 $K<I_{1}\left(\xi_{1}^{*}\right)$ 导致

$$
\varphi(0)=f\left(\xi_{1}^{*}\right)-f(0)-\beta_{2} \xi_{1}^{*}-K=I_{1}\left(\xi_{1}^{*}\right)-K>0 .
$$

另外, 我们可以计算得

$$
\begin{aligned}
& \varphi\left(\xi_{1}^{*}\right)=-K<0, \\
& \varphi^{\prime}(p)=\beta_{2}-f^{\prime}(p)<0 .
\end{aligned}
$$

因此, 存在唯一的解 $p_{1}^{*} \in\left(0, \xi_{1}^{*}\right)$ 满足 $\varphi\left(p_{1}^{*}\right)=0$, 即

$$
f\left(\xi_{1}^{*}\right)-f\left(p_{1}^{*}\right)-\beta_{2}\left(\xi_{1}^{*}-p_{1}^{*}\right)-K=0,
$$

等价地,

$$
\tilde{f}_{1}\left(\zeta_{1}^{*}\right)-\tilde{f}_{1}(0)-\beta_{2} \zeta_{1}^{*}-K=0,
$$

这里 $\zeta_{1}^{*}:=\xi_{1}^{*}-p_{1}^{*}$. 再利用 $\tilde{f}_{1}^{\prime}\left(\zeta_{1}^{*}\right)=f^{\prime}\left(\xi_{1}^{*}\right)=\beta_{2},(4.54)$ 可得

$$
\mathscr{M} \tilde{f}_{1}(0)=\max _{y \geqslant 0}\left\{\tilde{f}_{1}(y)-\beta_{2} y-K\right\}=\tilde{f}_{1}\left(\zeta_{1}^{*}\right)-\beta_{2} \zeta_{1}^{*}-K=\tilde{f}_{1}(0) .
$$

为简洁起见, 接下来不加证明地给出其他几种情形下的解. 
(ii) 当 $P<\frac{\lambda k_{3}}{\delta}\left[\theta_{2} h(m)+\left(\theta_{1}-\theta_{2}\right) \mu_{m}\right], f^{\prime}(0)=k_{3} \mathrm{e}^{\int_{0}^{x_{0}} \frac{\theta_{2}}{Q^{-1}(z)} d z}>k_{3}>\beta_{2}$ 且 $J\left(\xi_{2}^{*}\right)<K<I_{2}\left(\xi_{2}^{*}\right)$ 时, 定义一个可能的解

$$
\tilde{f}_{2}(x)=f\left(x+p_{2}^{*}\right)= \begin{cases}k_{3} \int_{0}^{x+p_{2}^{*}} \mathrm{e}^{\int_{y}^{x_{0}} \frac{\theta_{2}}{Q^{-1}(z)} d z} d y+P, & 0 \leqslant x<x_{0}-p_{2}^{*}, \\ k_{1} \mathrm{e}^{r_{+}\left(x+p_{2}^{*}-x_{0}\right)}+k_{2} \mathrm{e}^{r_{-}\left(x+p_{2}^{*}-x_{0}\right)}, & x_{0}-p_{2}^{*} \leqslant x \leqslant b_{2}, \\ \beta_{1}\left(x-b_{2}\right)+\tilde{f}_{2}\left(b_{2}\right), & x \geqslant b_{2},\end{cases}
$$

这里 $f(x)$ 同 (4.30), $b_{2}=b_{0}-p_{2}^{*}, b_{0}$ 由 (4.26) 给出, $p_{2}^{*} \in\left(0, x_{0}\right)$ 是下面方程的唯一解:

$$
f\left(\xi_{2}^{*}\right)-f\left(p_{2}^{*}\right)-\beta_{2}\left(\xi_{2}^{*}-p_{2}^{*}\right)-K_{2}=0,
$$

等价地,

$$
\tilde{f}_{2}\left(\zeta_{2}^{*}\right)-\tilde{f}_{2}(0)-\beta_{2} \zeta_{2}^{*}-K_{2}=0,
$$

这里 $\zeta_{2}^{*}:=\xi_{2}^{*}-p_{2}^{*}$. 相应地, 最优风险自留水平为

$$
\tilde{a}_{2}^{\pi^{*}}(x)=a^{\pi^{*}}\left(x+p_{2}^{*}\right)= \begin{cases}Q^{-1}\left(x+p_{2}^{*}\right), & 0 \leqslant x<x_{0}-p_{2}^{*}, \\ m, & x \geqslant x_{0}-p_{2}^{*},\end{cases}
$$

这里 $a^{\pi^{*}}(x)$ 由 (4.31) 给出. 那么, $\tilde{f}_{2}(x)$ 和 $\tilde{a}_{2}^{\pi^{*}}(x)$ 满足 (4.51)-(4.54).

(iii) 当 $P<\frac{\lambda k_{3}}{\delta}\left[\theta_{2} h(m)+\left(\theta_{1}-\theta_{2}\right) \mu_{m}\right], f^{\prime}(0)=k_{3} \mathrm{e}^{\int_{0}^{x_{0}} \frac{\theta_{2}}{Q^{-1}(z)} d z}>k_{3}>\beta_{2}$ 且 $0<K \leqslant J\left(\xi_{2}^{*}\right)$ 时, 定 义函数

$$
\tilde{f}_{3}(x)=f\left(x+p_{3}^{*}\right)= \begin{cases}k_{1} \mathrm{e}^{r_{+}\left(x+p_{3}^{*}-x_{0}\right)}+k_{2} \mathrm{e}^{r_{-}\left(x+p_{3}^{*}-x_{0}\right)}, & 0 \leqslant x \leqslant b_{3}, \\ \beta_{1}\left(x-b_{3}\right)+\tilde{f}_{3}\left(b_{3}\right), & x \geqslant b_{3},\end{cases}
$$

这里 $f(x)$ 由 (4.30) 给出, $b_{3}=b_{0}-p_{3}^{*}, b_{0}$ 由 (4.26) 给定, $p_{3}^{*} \in\left[x_{0}, \xi_{2}^{*}\right)$ 是如下方程的唯一解:

$$
f\left(\xi_{2}^{*}\right)-f\left(p_{3}^{*}\right)-\beta_{2}\left(\xi_{2}^{*}-p_{3}^{*}\right)-K=0,
$$

等价地,

$$
\tilde{f}_{3}\left(\zeta_{3}^{*}\right)-\tilde{f}_{3}(0)-\beta_{2} \zeta_{3}^{*}-K=0,
$$

这里 $\zeta_{3}^{*}:=\xi_{2}^{*}-p_{3}^{*}$. 相应地, 最优的风险自留水平是

$$
\tilde{a}_{3}^{\pi^{*}}(x)=a^{\pi^{*}}\left(x+p_{3}^{*}\right) \equiv m, \quad x \geqslant 0,
$$

这里 $a^{\pi^{*}}(x)$ 由 (4.31) 给出. 那么, $\tilde{f}_{3}(x)$ 和 $\tilde{a}_{3}^{\pi^{*}}(x)$ 满足 $(4.51)-(4.54)$.

(iv) 当 $\frac{\lambda k_{3}}{\delta}\left[\theta_{2} h(m)+\left(\theta_{1}-\theta_{2}\right) \mu_{m}\right] \leqslant P<\beta_{1} \lambda \theta_{1} \mu_{m} / \delta, f^{\prime}(0)=s_{1} r_{+}+s_{2} r_{-}>\beta_{2}$ 且 $K<I_{3}\left(\xi_{3}^{*}\right)$ 时, 定义函数

$$
\tilde{f}_{4}(x)=f\left(x+p_{4}^{*}\right)= \begin{cases}s_{1} \mathrm{e}^{r_{+}\left(x+p_{4}^{*}\right)}+s_{2} \mathrm{e}^{r_{-}\left(x+p_{4}^{*}\right)}, & 0 \leqslant x \leqslant b_{4}, \\ \beta_{1}\left(x-b_{4}\right)+\tilde{f}_{4}\left(b_{4}\right), & x \geqslant b_{4},\end{cases}
$$


这里 $f(x)$ 由 (4.42) 给出, $b_{4}=b_{0}-p_{4}^{*}, b_{0}$ 由 (4.45) 给出, $p_{4}^{*} \in\left(0, \xi_{3}^{*}\right)$ 是下面方程的唯一解:

$$
f\left(\xi_{3}^{*}\right)-f\left(p_{4}^{*}\right)-\beta_{2}\left(\xi_{3}^{*}-p_{4}^{*}\right)-K=0,
$$

等价地,

$$
\tilde{f}_{4}\left(\zeta_{4}^{*}\right)-\tilde{f}_{4}(0)-\beta_{2} \zeta_{4}^{*}-K=0,
$$

这里 $\zeta_{4}^{*}:=\xi_{3}^{*}-p_{4}^{*}$. 相应的最优风险自留水平为

$$
\tilde{a}_{4}^{\pi^{*}}(x) \equiv m, \quad x \geqslant 0 .
$$

那么, $\tilde{f}_{4}(x)$ 和 $\tilde{a}_{4}^{\pi^{*}}(x)$ 满足 (4.51)-(4.54). 另外, $p_{i}^{*}(i=1,2,3,4)$ 关于 $K$ 递减.

引理 4.3 由以上分析可知, $\tilde{f}_{i}(x)$ 和 $\tilde{a}_{i}^{\pi^{*}}(x)(i=1,2,3,4)$ 是 $(4.51)-(4.54)$ 的解, 并且 $\tilde{f}_{i}(x)$ 是二 阶连续可导且递增的凹函数, 其一阶导函数有界. 事实上, 它们也是 HJB 方程 (3.1) 和 (3.2) 的解. 主 要结论总结如下:

(1) 如果 $P<\frac{\lambda k_{3}}{\delta}\left[\theta_{2} h(m)+\left(\theta_{1}-\theta_{2}\right) \mu_{m}\right], f^{\prime}(0)=k_{3} \mathrm{e}^{\int_{0}^{x_{0}} \frac{\theta_{2}}{Q^{-1}(z)} d z}>\beta_{2} \geqslant k_{3}$ 且 $K<I_{1}\left(\xi_{1}^{*}\right)$, 则 $\tilde{f}_{1}(x)$ 和 $\tilde{a}_{1}^{\pi^{*}}(x)$ 的解可分别由 (4.55) 和 (4.56) 给出;

(2) 如果 $P<\frac{\lambda k_{3}}{\delta}\left[\theta_{2} h(m)+\left(\theta_{1}-\theta_{2}\right) \mu_{m}\right], f^{\prime}(0)=k_{3} \mathrm{e}^{\int_{0}^{x_{0}} \frac{\theta_{2}}{Q-1(z)} d z}>k_{3}>\beta_{2}$ 且 $J\left(\xi_{2}^{*}\right)<K<I_{2}\left(\xi_{2}^{*}\right)$, 则 $\tilde{f}_{2}(x)$ 和 $\tilde{a}_{2}^{\pi^{*}}(x)$ 的解可分别由 (4.58) 和 (4.61) 给出;

(3) 如果 $P<\frac{\lambda k_{3}}{\delta}\left[\theta_{2} h(m)+\left(\theta_{1}-\theta_{2}\right) \mu_{m}\right], f^{\prime}(0)=k_{3} \mathrm{e}^{\int_{0}^{x_{0}} \frac{\theta_{2}}{Q-1(z)} d z}>k_{3}>\beta_{2}$ 且 $0<K \leqslant J\left(\xi_{2}^{*}\right)$, 则 $\tilde{f}_{3}(x)$ 和 $\tilde{a}_{3}^{\pi^{*}}(x)$ 的解可分别由 (4.62) 和 (4.65) 给出;

(4) 如果 $\frac{\lambda k_{3}}{\delta}\left[\theta_{2} h(m)+\left(\theta_{1}-\theta_{2}\right) \mu_{m}\right] \leqslant P<\beta_{1} \lambda \theta_{1} \mu_{m} / \delta, f^{\prime}(0)=s_{1} r_{+}+s_{2} r_{-}>\beta_{2}$ 且 $K<I_{3}\left(\xi_{3}^{*}\right)$, 则 $\tilde{f}_{4}(x)$ 和 $\tilde{a}_{4}^{\pi^{*}}(x)$ 的解可分别由 (4.66) 和 (4.69) 给出.

引理 4.3 的证明方法与引理 4.2 类似, 故省略.

\section{3 值函数与最优策略}

在第 4.1 和 4.2 小节中, 我们分别考虑了两种特殊情形: 一种是自始至终不注资, 另一种是利用 注资永不破产. 由于模型的 Markov 性, 这两种情形必有一种最优, 其最优性依赖于模型参数之间的 关系.

以下表述中所言的参数为 $b$ 的 Barrier 分红策略是指盈余一旦超过 $b \geqslant 0$ 则超出的部分立即作为 红利分发给股东, 否则不予分红. 该策略下到 $t$ 时刻为止的累积分红额可记为

$$
D_{t}^{\pi^{*}}(b)=(x-b)_{+}+\int_{0}^{t} I_{\left\{X_{s}^{\pi^{*}} \geqslant b\right\}} d D_{s}^{\pi^{*}}(b) .
$$

定理 4.1 当 (4.1) 成立时, 我们可在 10 种不同情形下给出了值函数 $V(x)$ 和最优策略 $\pi^{*}$ 的解, 它们包含了 (4.1) 下的所有可能.

情形 $1 P<\frac{\lambda k_{3}}{\delta}\left[\theta_{2} h(m)+\left(\theta_{1}-\theta_{2}\right) \mu_{m}\right]$ 且 $\beta_{1}<f^{\prime}(0)=k_{3} \mathrm{e}^{\int_{0}^{x_{0}} \frac{\theta_{2}}{Q^{-1}(z)} d z} \leqslant \beta_{2}$.

此时由 (4.26) 给定参数 $b_{0}$, 那么, 值函数 $V(x)$ 等于 (4.30) 中定义的 $f(x)$. 最优再保险策略由 (4.31) 中 $a^{\pi^{*}}$ 确定; 参数为 $b_{0}$ 的 Barrier 策略是最优的分红策略, 即 $D^{\pi^{*}}=\left\{D_{t}^{\pi^{*}}\left(b_{0}\right), t \geqslant 0\right\}$; 始终不注资是 最优的策略, 即 $R_{t}^{\pi^{*}} \equiv 0$. 从而, $t>0$ 时策略 $\pi^{*}=\left(a^{\pi^{*}} ; D^{\pi^{*}} ; R^{\pi^{*}}\right)$ 控制下的盈余过程可表示为

$$
\left\{\begin{array}{l}
X_{t}^{\pi^{*}}=x+\int_{0}^{t}\left[\left(\theta_{1}-\theta_{2}\right) \lambda \mu_{m}+\theta_{2} \lambda \mu\left(a^{\pi^{*}}\left(X_{s}^{\pi^{*}}\right)\right)\right] d s+\int_{0}^{t} \sqrt{\lambda \nu\left(a^{\pi^{*}}\left(X_{s}^{\pi^{*}}\right)\right)} d B_{s}-D_{t}^{\pi^{*}}\left(b_{0}\right), \\
X_{t}^{\pi^{*}} \leqslant b_{0} .
\end{array}\right.
$$


情形 $2 P<\frac{\lambda k_{3}}{\delta}\left[\theta_{2} h(m)+\left(\theta_{1}-\theta_{2}\right) \mu_{m}\right], f^{\prime}(0)=k_{3} \mathrm{e}^{\int_{0}^{x_{0}} \frac{\theta_{2}}{Q^{-1}(z)} d z}>\beta_{2} \geqslant k_{3}$ 且 $K \geqslant I_{1}\left(\xi_{1}^{*}\right)$. 值函数 $V(x)$ 和相应的最优策略 $\pi^{*}$ 与情形 1 中相同.

情形 $3 P<\frac{\lambda k_{3}}{\delta}\left[\theta_{2} h(m)+\left(\theta_{1}-\theta_{2}\right) \mu_{m}\right], f^{\prime}(0)=k_{3} \mathrm{e}^{\int_{0}^{x_{0}} \frac{\theta_{2}}{Q^{-1}(z)} d z}>k_{3}>\beta_{2}$ 且 $K \geqslant I_{2}\left(\xi_{2}^{*}\right)$.

值函数 $V(x)$ 和相应的最优策略 $\pi^{*}$ 与情形 1 中相同.

情形 $4 \frac{\lambda k_{3}}{\delta}\left[\theta_{2} h(m)+\left(\theta_{1}-\theta_{2}\right) \mu_{m}\right] \leqslant P<\beta_{1} \lambda \theta_{1} \mu_{m} / \delta$ 和 $f^{\prime}(0)=\frac{\beta_{1} r_{-}}{r_{-}-r_{+}} \mathrm{e}^{-r_{+} b_{1}}+\frac{\beta_{1} r_{+}}{r_{+}-r_{-}} \mathrm{e}^{-r_{-} b_{1}} \leqslant \beta_{2}$. 此时由 (4.45) 给定参数 $b_{0}$, 那么, 值函数 $V(x)$ 与 (4.42) 中给定的 $f(x)$ 相同. 保险公司始终不购 买再保险为最优, 即 $a^{\pi^{*}} \equiv m$; 参数为 $b_{0}$ 的 Barrier 策略是最优的分红策略, 即 $D^{\pi^{*}}=\left\{D_{t}^{\pi^{*}}\left(b_{0}\right), t \geqslant 0\right\}$; 始终不注资是最优的策略, 即 $R_{t}^{\pi^{*}} \equiv 0$. 从而, 当 $t>0$ 时, 策略 $\pi^{*}=\left(a^{\pi^{*}} ; D^{\pi^{*}} ; R^{\pi^{*}}\right)$ 控制下的盈余过 程可表示为

$$
\left\{\begin{array}{l}
X_{t}^{\pi^{*}}=x+\theta_{1} \lambda \mu_{m} t+\sqrt{\lambda \nu_{m}} B_{t}-D_{t}^{\pi^{*}}\left(b_{0}\right), \\
X_{t}^{\pi^{*}} \leqslant b_{0} .
\end{array}\right.
$$

情形 $5 \frac{\lambda k_{3}}{\delta}\left[\theta_{2} h(m)+\left(\theta_{1}-\theta_{2}\right) \mu_{m}\right] \leqslant P<\beta_{1} \lambda \theta_{1} \mu_{m} / \delta, f^{\prime}(0)=s_{1} r_{+}+s_{2} r_{-}>\beta_{2}$ 和 $K \geqslant I_{3}\left(\xi_{3}^{*}\right)$. 此时值函数 $V(x)$ 和相应的最优策略 $\pi^{*}$ 与情形 4 中相同.

情形 $6 \quad P \geqslant \beta_{1} \lambda \theta_{1} \mu_{m} / \delta$.

值函数 $V(x)$ 与 $(4.50)$ 中的 $f(x)$ 相等. 这意味着最优的策略是把当前所有盈余立即作为红利分 发给股东, 并宣告破产获得终端值, 即 $a^{\pi^{*}} \equiv m, D_{t}^{\pi^{*}} \equiv x$ 且 $R_{t}^{\pi^{*}} \equiv 0$.

情形 $7 \quad P<\frac{\lambda k_{3}}{\delta}\left[\theta_{2} h(m)+\left(\theta_{1}-\theta_{2}\right) \mu_{m}\right], f^{\prime}(0)=k_{3} \mathrm{e}^{\int_{0}^{x_{0}} \frac{\theta_{2}}{Q^{-1}(z)} d z}>\beta_{2} \geqslant k_{3}$ 且 $K<I_{1}\left(\xi_{1}^{*}\right)$.

由 (4.26) 给定 $b_{0}$ 且 $b_{1}=b_{0}-p_{1}^{*}$, 此时值函数 $V(x)$ 与 $(4.55)$ 中的 $\tilde{f}_{1}(x)$ 相等. 最优的再保险策略 由 (4.56) 中的 $\tilde{a}_{1}^{\pi^{*}}$ 决定; 参数为 $b_{1}$ 的 Barrier 策略是最优的分红策略, 即 $D^{\pi^{*}}=\left\{D_{t}^{\pi^{*}}\left(b_{1}\right), t \geqslant 0\right\}$; 当 且仅当盈余为 0 时进行注资是最优的, 且最优的注资额为 $\zeta_{1}^{*}$, 即最优注资策略 $R^{\pi^{*}}=\left\{R_{t}^{\pi^{*}}, t \geqslant 0\right\}$ 可 表示为

$$
\left\{\begin{array}{l}
\int_{0}^{\infty} I_{\left\{t: X_{t}^{\pi^{*}}>0\right\}} d R_{t}^{\pi^{*}}=0, \\
\tau_{1}^{\pi^{*}}=\inf \left\{t \geqslant 0: X_{t-}^{\pi^{*}}=0\right\} \\
\tau_{i}^{\pi^{*}}=\inf \left\{t>\tau_{n-1}^{\pi^{*}}: X_{t-}^{\pi^{*}}=0\right\}, \quad n=2,3, \ldots
\end{array}\right.
$$

且

$$
\eta_{i}^{\pi^{*}} \equiv \zeta_{1}^{*}=\xi_{1}^{*}-p_{1}^{*}, \quad i=1,2, \ldots
$$

相应地, 当 $t>0$ 时, 最优策略 $\pi^{*}=\left(\tilde{a}_{1}^{\pi^{*}} ; D^{\pi^{*}} ; R^{\pi^{*}}\right)$ 控制下的盈余过程可表示为

$$
\left\{\begin{aligned}
& X_{t}^{\pi^{*}}= x+\int_{0}^{t}\left[\left(\theta_{1}-\theta_{2}\right) \lambda \mu_{m}+\theta_{2} \lambda \mu\left(\tilde{a}_{1}^{\pi^{*}}\left(X_{s}^{\pi^{*}}\right)\right)\right] d s+\int_{0}^{t} \sqrt{\lambda \nu\left(\tilde{a}_{1}^{\pi^{*}}\left(X_{s}^{\pi^{*}}\right)\right)} d B_{s} \\
&+\sum_{i=1}^{\infty} I_{\left\{\tau_{i}^{\pi^{*}} \leqslant t\right\}} \eta_{i}^{\pi^{*}}-D_{t}^{\pi^{*}}\left(b_{1}\right), \\
& 0 \leqslant X_{t}^{\pi^{*}} \leqslant b_{1} .
\end{aligned}\right.
$$

情形 $8 \quad P<\frac{\lambda k_{3}}{\delta}\left[\theta_{2} h(m)+\left(\theta_{1}-\theta_{2}\right) \mu_{m}\right], f^{\prime}(0)=k_{3} \mathrm{e}^{\int_{0}^{x_{0}} \frac{\theta_{2}}{Q^{-1}(z)} d z}>k_{3}>\beta_{2}$ 且 $J\left(\xi_{2}^{*}\right)<K<I_{2}\left(\xi_{2}^{*}\right)$. 
由 (4.26) 给定 $b_{0}$ 且 $b_{2}=b_{0}-p_{2}^{*}$. 值函数 $V(x)$ 与 $(4.58)$ 中的 $\tilde{f}_{2}(x)$ 相等. 最优再保险策略 $\tilde{a}_{2}^{\pi^{*}}$ 由 (4.61) 确定; 参数为 $b_{2}$ 的 Barrier 策略是最优的分红策略, 即 $D^{\pi^{*}}=\left\{D_{t}^{\pi^{*}}\left(b_{2}\right), t \geqslant 0\right\}$; 当且仅当公 司盈余为 0 时注资最优, 且注资额为 $\zeta_{2}^{*}$, 即最优注资过程 $R^{\pi^{*}}=\left\{R_{t}^{\pi^{*}}, t \geqslant 0\right\}$ 可由 (4.73) 和

$$
\eta_{i}^{\pi^{*}} \equiv \zeta_{2}^{*}=\xi_{2}^{*}-p_{2}^{*}, \quad i=1,2, \ldots
$$

表示. 相应地, $t>0$ 时最优策略 $\pi^{*}=\left(\tilde{a}_{2}^{\pi^{*}} ; D^{\pi^{*}} ; R^{\pi^{*}}\right)$ 控制下的盈余过程可表示为

$$
\left\{\begin{aligned}
X_{t}^{\pi^{*}}= & x+\int_{0}^{t}\left[\left(\theta_{1}-\theta_{2}\right) \lambda \mu_{m}+\theta_{2} \lambda \mu\left(\tilde{a}_{2}^{\pi^{*}}\left(X_{s}^{\pi^{*}}\right)\right)\right] d s+\int_{0}^{t} \sqrt{\lambda \nu\left(\tilde{a}_{2}^{\pi^{*}}\left(X_{s}^{\pi^{*}}\right)\right)} d B_{s} \\
& +\sum_{i=1}^{\infty} I_{\left\{\tau_{i}^{\pi^{*}} \leqslant t\right\}} \eta_{i}^{\pi^{*}}-D_{t}^{\pi^{*}}\left(b_{2}\right), \\
0 \leqslant X_{t}^{\pi^{*}} \leqslant b_{2} . &
\end{aligned}\right.
$$

情形 $9 P<\frac{\lambda k_{3}}{\delta}\left[\theta_{2} h(m)+\left(\theta_{1}-\theta_{2}\right) \mu_{m}\right], f^{\prime}(0)=k_{3} \mathrm{e}^{\int_{0}^{x_{0}} \frac{\theta_{2}}{Q^{-1}(z)} d z}>k_{3}>\beta_{2}$ 且 $0<K \leqslant J\left(\xi_{2}^{*}\right)$.

由 (4.26) 给定 $b_{0}$ 且 $b_{3}=b_{1}-p_{3}^{*}$, 此时值函数 $V(x)$ 等于 $(4.62)$ 中的 $\tilde{f}_{3}(x)$. 不购买再保险是最优 的选择, 即 $\tilde{a}_{3}^{\pi^{*}}(x) \equiv m$; 参数为 $b_{3}$ 的 Barrier 策略是最优的分红策略, 即 $D^{\pi^{*}}=\left\{D_{t}^{\pi^{*}}\left(b_{3}\right), t \geqslant 0\right\}$; 当且 仅当公司盈余为 0 时注资最优, 且注资额为 $\zeta_{3}^{*}$, 即最优注资策略 $R^{\pi^{*}}=\left\{R_{t}^{\pi^{*}}, t \geqslant 0\right\}$ 可由 (4.73) 和

$$
\eta_{i}^{\pi^{*}} \equiv \zeta_{3}^{*}=\xi_{2}^{*}-p_{3}^{*}, \quad i=1,2, \ldots
$$

表示. 相应地, 当 $t>0$ 时, 最优策略 $\pi^{*}=\left(\tilde{a}_{2}^{\pi^{*}} ; D^{\pi^{*}} ; R^{\pi^{*}}\right)$ 控制下的盈余过程可表示为

$$
\left\{\begin{aligned}
& X_{t}^{\pi^{*}}= x+\int_{0}^{t}\left[\left(\theta_{1}-\theta_{2}\right) \lambda \mu_{m}+\theta_{2} \lambda \mu\left(\tilde{a}_{3}^{\pi^{*}}\left(X_{s}^{\pi^{*}}\right)\right)\right] d s+\int_{0}^{t} \sqrt{\lambda \nu\left(\tilde{a}_{3}^{\pi^{*}}\left(X_{s}^{\pi^{*}}\right)\right)} d B_{s} \\
&+\sum_{i=1}^{\infty} I_{\left\{\tau_{i}^{\pi^{*}} \leqslant t\right\}} \eta_{i}^{\pi^{*}}-D_{t}^{\pi^{*}}\left(b_{3}\right), \\
& 0 \leqslant X_{t}^{\pi^{*}} \leqslant b_{3} .
\end{aligned}\right.
$$

情形 $10 \frac{\lambda k_{3}}{\delta}\left[\theta_{2} h(m)+\left(\theta_{1}-\theta_{2}\right) \mu_{m}\right] \leqslant P<\beta_{1} \lambda \theta_{1} \mu_{m} / \delta, f^{\prime}(0)=s_{1} r_{+}+s_{2} r_{-}>\beta_{2}$ 且 $0<K<I_{3}\left(\xi_{3}\right)$.

由 (4.45) 给定 $b_{0}$ 且 $b_{4}=b_{0}-p_{4}^{*}$, 此时值函数 $V(x)$ 等于 (4.66) 中的 $\tilde{f}_{4}(x)$. 不购买任何再保险是 最优的选择, 即 $\tilde{a}_{4}^{\pi^{*}} \equiv m$; 参数为 $b_{4}$ 的 Barrier 策略 $D^{\pi^{*}}=\left\{D_{t}^{\pi^{*}}\left(b_{4}\right), t \geqslant 0\right\}$ 是最优的分红策略; 当且 仅当公司盈余为 0 时注资最优, 且注资额为 $\zeta_{4}^{*}$; 最优注资策略 $R^{\pi^{*}}=\left\{R_{t}^{\pi^{*}}, t \geqslant 0\right\}$ 可由 (4.73) 和

$$
\eta_{i}^{\pi^{*}} \equiv \zeta_{4}^{*}=\xi_{3}^{*}-p_{4}^{*}, \quad i=1,2, \ldots
$$

表示. 相应地, $t>0$ 时最优策略 $\pi^{*}=\left(\tilde{a}_{4}^{\pi^{*}} ; D^{\pi^{*}} ; R^{\pi^{*}}\right)$ 控制下盈余过程可表示为

$$
\left\{\begin{array}{l}
X_{t}^{\pi^{*}}=x+\theta_{1} \lambda \mu_{m} t+\sqrt{\lambda \nu_{m}} B_{t}+\sum_{i=1}^{\infty} I_{\left\{\tau_{i}^{\pi^{*}} \leqslant t\right\}} \eta_{i}^{\pi^{*}}-D_{t}^{\pi^{*}}\left(b_{4}\right), \\
0 \leqslant X_{t}^{\pi^{*}} \leqslant b_{4} .
\end{array}\right.
$$

引理 4.1 和 4.2 已经证明了以上给出的 $V(x)$ 表达式均满足 HJB 方程 (3.1) 和 (3.2), 它们都是二 阶连续可导且递增的凹函数, 且一阶导函数有界. 另外, 我们能够证明上述 10 种情形下给出的策略 $\pi^{*}$ 满足 $V(x)=V\left(x, \pi^{*}\right)$. 所以, 再利用定理 3.1 的结论, 本定理即可得证. 附录 D 给出情形 7 的证明过 程, 其他情形可类似证明. 


\section{5 第二种情形: $\theta_{2} \in\left[\frac{m}{\lambda \nu_{m}}\left(\lambda \theta_{1} \mu_{m}+\sqrt{\left(\lambda \theta_{1} \mu_{m}\right)^{2}+2 \lambda \delta \nu_{m}}\right), \infty\right)$}

本节研究

$$
\theta_{2} \geqslant \frac{m}{\lambda \nu_{m}}\left(\lambda \theta_{1} \mu_{m}+\sqrt{\left(\lambda \theta_{1} \mu_{m}\right)^{2}+2 \lambda \delta \nu_{m}}\right)
$$

的情形. 此时可认为再保险人安全负荷过大, 再保险合约索价过高, 保险公司应放弃购买再保险. 与第 4 节类似, 我们需要首先考虑两种特殊情形下的最优化问题, 它们各自对应不同的边界条件. 然后证明问 题 2.1 的解必然与这两种特殊情形之一的解相同. 本节中结论的证明类似于第 4 节, 故省略了大部分 证明过程.

\section{1 不选择注资的情形}

类似于第 4.1 小节, 本节研究边界条件为 $v(0)=P$ 且 $\mathscr{M} v(0)-v(0) \leqslant 0$ 的情形. 这意味着注资不 是最优的选择, 一旦公司盈余为 0 就立即宣布破产退出市场. 那么, HJB 方程中 $v(x)$ 的解 $g(x)$ 应该 满足

$$
\begin{aligned}
& \max _{0 \leqslant a \leqslant m}\left\{\mathscr{A}^{a} g(x)\right\}=0, \quad 0 \leqslant x \leqslant d_{0}, \\
& \beta_{1}-g^{\prime}(x)=0, \quad x \geqslant d_{0}, \\
& g(0)=P \\
& \mathscr{M} g(0)-g(0) \leqslant 0,
\end{aligned}
$$

这里分红临界点 $d_{0} \geqslant 0$ 待定. 在 (5.1) 前提条件下, 我们猜想最优的再保险策略是 $a^{\pi^{*}} \equiv m$, 即始终不 购买再保险. 那么, (5.2) 和 (5.3) 有如下二次连续可导且递增的凹解:

$$
g(x)= \begin{cases}l_{1} \mathrm{e}^{r_{+} x}+l_{2} \mathrm{e}^{r_{-} x}, & 0 \leqslant x \leqslant d_{0}, \\ \beta_{1}\left(x-d_{0}\right)+g\left(d_{0}\right), & x \geqslant d_{0} .\end{cases}
$$

利用该函数在 $d_{0}$ 处的光滑条件可得

$$
\begin{aligned}
& l_{1}=\frac{\beta_{1} r_{-}}{r_{+}\left(r_{-}-r_{+}\right)} \mathrm{e}^{-r_{+} d_{0}}, \\
& l_{2}=\frac{\beta_{1} r_{+}}{r_{-}\left(r_{+}-r_{-}\right)} \mathrm{e}^{-r_{-} d_{0}} .
\end{aligned}
$$

边界条件 (5.4) 可得到参数 $d_{0}$ 满足的方程, 即

$$
g(0)=\frac{\beta_{1} r_{-}}{r_{+}\left(r_{-}-r_{+}\right)} \mathrm{e}^{-r_{+} d_{0}}+\frac{\beta_{1} r_{+}}{r_{-}\left(r_{+}-r_{-}\right)} \mathrm{e}^{-r_{-} d_{0}}=P .
$$

类似于 (4.45), 当且仅当 $P<\beta_{1} \lambda \theta_{1} \mu_{m} / \delta$ 时, 方程 (5.9) 存在唯一解 $d_{0}>0$. 为了证明 $a(x) \equiv m$ 是最 优的, 考虑到 (4.6), 我们只要证明对任意 $x \in\left(0, d_{0}\right)$ 有

$$
G(x):=-\theta_{2} \frac{g^{\prime}(x)}{g^{\prime \prime}(x)}=\theta_{2} \frac{-r_{-} \mathrm{e}^{-r_{+}\left(d_{0}-x\right)}+r_{+} \mathrm{e}^{-r_{-}\left(d_{0}-x\right)}}{r_{+} r_{-}\left(\mathrm{e}^{-r_{+}\left(d_{0}-x\right)}-\mathrm{e}^{-r_{-}\left(d_{0}-x\right)}\right)} \geqslant m
$$

986 
成立即可. 因为 $G(0)>m$ 且

$$
G^{\prime}(x)=\frac{-\theta_{2}\left(r_{+}-r_{-}\right)^{2}}{\left(r_{+} r_{-}\right)^{3}\left(\mathrm{e}^{-r_{+}\left(d_{0}-x\right)}-\mathrm{e}^{-r_{-}\left(d_{0}-x\right)}\right)^{2}} \mathrm{e}^{-\left(r_{+}+r_{-}\right)\left(d_{0}-x\right)}>0,
$$

所以, 不等式 (5.10) 成立.

可以证明 $g^{\prime}(0)=\frac{\beta_{1}}{r_{+}-r_{-}}\left(r_{+} \mathrm{e}^{-r_{-} d_{0}}-r_{-} \mathrm{e}^{-r_{+} d_{0}}\right)>\beta_{1}$ 成立. 我们根据 $g^{\prime}(0)$ 与 $\beta_{2}$ 之间的大小关系 讨论 (5.5) 的成立条件.

(i) 当 $\beta_{1}<g^{\prime}(0) \leqslant \beta_{2}$ 时, 由于 $g^{\prime}(x)$ 在区间 $[0, \infty)$ 上递减，故 $g^{\prime}(x) \leqslant \beta_{2}$ 恒成立. 因此, $\mathscr{M} g(0)-g(0)=-K<0$, 即 $(5.5)$ 成立.

(ii) 当 $g^{\prime}(0)>\beta_{2}$ 时, 存在唯一的根 $\rho^{*} \in\left(0, d_{1}\right)$ 满足方程 $g^{\prime}\left(\rho^{*}\right)=\beta_{2}$. 定义

$$
H\left(\rho^{*}\right):=\int_{0}^{\rho^{*}}\left(g^{\prime}(x)-\beta_{2}\right) d x=g\left(\rho^{*}\right)-g(0)-\beta_{2} \rho^{*},
$$

这里 $g(x)$ 由 (5.6) 给定. 那么, 当且仅当

$$
K \geqslant H\left(\rho^{*}\right)
$$

时 (5.5) 成立, 我们将在下一节中讨论相反的情形.

如果 $P \geqslant \beta_{1} \lambda \theta_{1} \mu_{m} / \delta$, 那么 (5.9) 中的 $d_{0}$ 不存在, 方程 (5.2)-(5.5) 的解可表示为

$$
g(x)=\beta_{1} x+P, \quad x \geqslant 0 .
$$

引理 5.1 通过以上分析, 我们得到了 (5.2)-(5.5) 中 $g(x)$ 的解以及相应的最优风险自留水平 $a^{\pi^{*}}(x)$, 并且 $g(x)$ 是二阶连续可导且递增的凹函数, 其一阶导函数有界. 事实上, 它们也满足 HJB 方 程 (3.1) 和 (3.2). 主要结论总结如下:

(1) 如果 $P<\beta_{1} \lambda \theta_{1} \mu_{m} / \delta$ 且 $\beta_{1}<g^{\prime}(0)=\frac{\beta_{1}}{r_{+}-r_{-}}\left(r_{+} \mathrm{e}^{-r_{-} d_{0}}-r_{-} \mathrm{e}^{-r_{+} d_{0}}\right) \leqslant \beta_{2}$, 则 $g(x)$ 的解如 (5.6) 所示, 且 $a^{\pi^{*}}(x) \equiv m$;

(2) 如果 $P<\beta_{1} \lambda \theta_{1} \mu_{m} / \delta, g^{\prime}(0)=\frac{\beta_{1}}{r_{+}-r_{-}}\left(r_{+} \mathrm{e}^{-r_{-} d_{0}}-r_{-} \mathrm{e}^{-r_{+} d_{0}}\right)>\beta_{2}$ 且 $K \geqslant H\left(\rho^{*}\right)$, 则 $g(x)$ 的解 如 (5.6) 所示, 且 $a^{\pi^{*}}(x) \equiv m$

(3) 如果 $P \geqslant \beta_{1} \lambda \theta_{1} \mu_{m} / \delta$, 则 $g(x)$ 的解如 (5.14) 所示, 且 $a^{\pi^{*}}(x) \equiv m$.

引理 5.1 的证明方法与引理 4.2 类似, 此处省略.

\section{2 不允许破产的情形}

当 $P<\beta_{1} \lambda \theta_{1} \mu_{m} / \delta, g^{\prime}(0)=\frac{\beta_{1}}{r_{+}-r_{-}}\left(r_{+} \mathrm{e}^{-r_{-} d_{0}}-r_{-} \mathrm{e}^{-r_{+} d_{0}}\right)>\beta_{2}$ 且 $K<H\left(\rho^{*}\right)$ 时, 方程组 (5.2)-(5.5) 不存在合适的解. 换句话讲, 当盈余为 0 时破产不再是最优的选择, 公司应当采取注资手段避免破产. 相应地, HJB 方程中 $v(x)$ 的解 $\tilde{g}(x)$ 应该满足

$$
\begin{aligned}
& \max _{0 \leqslant \tilde{a} \leqslant m}\left\{\mathscr{A}^{\tilde{a}} \tilde{g}(x)\right\}=0, \quad 0 \leqslant x \leqslant \tilde{d}_{0}, \\
& \beta_{1}-\tilde{g}(x)=0, \quad x \geqslant \tilde{d}_{0}, \\
& \mathscr{M} \tilde{g}(0)-\tilde{g}(0)=0, \\
& \tilde{g}(0) \geqslant P
\end{aligned}
$$


其中分红临界点 $\tilde{d}_{0} \geqslant 0$ 待定. 利用第 4.2 小节中相同的方法, 我们可求解方程组 (5.15)-(5.18). 设 $g(x)$ 和 $d_{0}$ 分别由 (5.6) 和 (5.9) 给出. 令 $q^{*} \in\left(0, \rho^{*}\right)$ 为方程

$$
g\left(\rho^{*}\right)-g\left(q^{*}\right)-\beta_{2}\left(\rho^{*}-q^{*}\right)-K=0
$$

的唯一解, 且 $\tilde{d}_{0}=d_{1}=d_{0}-q^{*}$, 那么, 我们可以证明

$$
\tilde{g}(x)=g\left(x+q^{*}\right)= \begin{cases}l_{1} \mathrm{e}^{r_{+}\left(x+q^{*}\right)}+l_{2} \mathrm{e}^{r_{-}\left(x+q^{*}\right)}, & 0 \leqslant x \leqslant d_{1}, \\ \beta_{1}\left(x-d_{1}\right)+\tilde{g}\left(d_{1}\right), & x \geqslant d_{1}\end{cases}
$$

是 (5.15) - (5.18) 的合适解且 $\tilde{a}^{\pi^{*}}(x) \equiv m$ 是最优的风险自留水平.

引理 5.2 当 $P<\beta_{1} \lambda \theta_{1} \mu_{m} / \delta, g^{\prime}(0)=\frac{\beta_{1}}{r_{+}-r_{-}}\left(r_{+} \mathrm{e}^{-r_{-} d_{0}}-r_{-} \mathrm{e}^{-r_{+} d_{0}}\right)>\beta_{2}$ 且 $K<H\left(\rho^{*}\right)$ 时, (5.19) 中 $\tilde{g}(x)$ 和 $a^{\pi^{*}}(x) \equiv m$ 是方程组 (5.15)-(5.18) 的解, 且 $\tilde{g}(x)$ 是二阶连续可导且递增的凹函数, 其一阶 导函数有界.

利用引理 4.2 中的方法可证引理 5.2 结论, 具体过程省略.

\section{3 值函数和最优策略}

在第 5.1 和 5.2 小节分析的基础上, 本节给出问题 2.1 在条件 (5.1) 下的显示解.

定理 5.1 在 (5.1) 的前提下, 值函数 $V(x)$ 和最优策略 $\pi^{*}$ 可在如下 4 种情形下给出, 它们包括 了 (5.1) 下的所有可能.

情形 $1 P<\beta_{1} \lambda \theta_{1} \mu_{m} / \delta$ 且 $\beta_{1}<g^{\prime}(0)=\frac{\beta_{1}}{r_{+}-r_{-}}\left(r_{+} \mathrm{e}^{-r_{-} d_{0}}-r_{-} \mathrm{e}^{-r_{+} d_{0}}\right) \leqslant \beta_{2}$.

由 (5.9) 给定参数 $d_{0}$. 此时值函数 $V(x)$ 与 $(5.6)$ 中 $g(x)$ 相等. 参数为 $d_{0}$ 的 Barrier 策略是最优 的分红策略, 即 $D^{\pi^{*}}=\left\{D_{t}^{\pi^{*}}\left(d_{0}\right), t \geqslant 0\right\}$. 不注资和不购买再保险是最优的, 即 $R_{t}^{\pi^{*}} \equiv 0$ 和 $a^{\pi^{*}}(x) \equiv m$. 在 $t>0$ 时最优策略 $\pi^{*}=\left(a^{\pi^{*}} ; D^{\pi^{*}} ; R^{\pi^{*}}\right)$ 控制下的盈余过程可表示为

$$
\left\{\begin{array}{l}
X_{t}^{\pi^{*}}=x+\lambda \theta_{1} \mu_{m} t+\sqrt{\lambda \nu_{m}} B_{t}-D_{t}^{\pi^{*}}\left(d_{0}\right) \\
X_{t}^{\pi^{*}} \leqslant d_{0}
\end{array}\right.
$$

情形 $2 P<\beta_{1} \lambda \theta_{1} \mu_{m} / \delta, g^{\prime}(0)=\frac{\beta_{1}}{r_{+}-r_{-}}\left(r_{+} \mathrm{e}^{-r_{-} d_{0}}-r_{-} \mathrm{e}^{-r_{+} d_{0}}\right)>\beta_{2}$ 且 $K \geqslant H\left(\rho^{*}\right)$.

值函数 $V(x)$ 和最优策略 $\pi^{*}$ 与情形 1 相同.

情形 $3 P<\beta_{1} \lambda \theta_{1} \mu_{m} / \delta, g^{\prime}(0)=\frac{\beta_{1}}{r_{+}-r_{-}}\left(r_{+} \mathrm{e}^{-r_{-} d_{0}}-r_{-} \mathrm{e}^{-r_{+} d_{0}}\right)>\beta_{2}$ 且 $0<K<H\left(\rho^{*}\right)$.

值函数 $V(x)$ 与 $(5.19)$ 中 $\tilde{g}(x)$ 相等. 不购买再保险是最优的, 即 $\tilde{a}^{\pi^{*}}(x) \equiv m$; 参数为 $d_{1}$ 的 Barrier 策略 $D^{\pi^{*}}=\left\{D_{t}^{\pi^{*}}\left(d_{1}\right), t \geqslant 0\right\}$ 是最优的分红策略; 当且仅当盈余为 0 时注资最优, 且注资额为 $\varrho^{*}$, 即 $R^{\pi^{*}}$ 可由 (4.73) 和

$$
\eta_{i}^{\pi^{*}} \equiv \varrho^{*}:=\rho^{*}-q^{*}, \quad i=1,2, \ldots
$$

给定. 在 $t>0$ 时最优策略 $\pi^{*}=\left(\tilde{a}^{\pi^{*}} ; D^{\pi^{*}} ; R^{\pi^{*}}\right)$ 控制下的盈余过程可表示为

$$
\left\{\begin{array}{l}
X_{t}^{\pi^{*}}=x+\lambda \theta_{1} \mu_{m} t+\sqrt{\lambda \nu_{m}} B_{t}+\sum_{i=1}^{\infty} I_{\left\{\tau_{i}^{\pi^{*}} \leqslant t\right\}} \eta_{i}^{\pi^{*}}-D_{t}^{\pi^{*}}\left(d_{1}\right), \\
0 \leqslant X_{t}^{\pi^{*}} \leqslant d_{1} .
\end{array}\right.
$$


情形 $4 P \geqslant \beta_{1} \lambda \theta_{1} \mu_{m} / \delta$.

此时保险公司立即将所有盈余分发给股东并宣告破产是最优的选择, 值函数和 $V(x)$ 和 $a^{\pi^{*}}$ 与定 理 4.1 中的情形 6 相同.

证明方法与定理 4.1 相同, 证明过程省略.

注 5.1 在保险实务中, 保险金额和理赔额都是有限的, 从这个角度来看, 本文中 $m<\infty$ 的假设 合理. 出于数学上的兴趣, 我们也可以讨论 $m=\infty$ 的情形, 那么,

$$
\frac{m}{\lambda \nu_{m}}\left(\lambda \theta_{1} \mu_{m}+\sqrt{\left(\lambda \theta_{1} \mu_{m}\right)^{2}+2 \lambda \delta \nu_{m}}\right)=\infty .
$$

相应地, 第 5 节讨论的情形不存在, 而第 4 节将退化为 $m \rightarrow \infty$ 时的情形. 类似讨论可参见文献 [3,23].

\section{6 结论}

本文在扩散模型下考虑了一个最优分红、注资和溢额再保险问题. 与以往文献相比, 本文的主要 贡献是将注资考虑在内并分析了交易费用和任意破产终端值 $P$ 对最优策略的影响. 研究结果表明, 盈 余增加时保险公司应减少再保险购买量; 公司盈余足够大或者再保险成本过高时不应购买最保险; 当 交易费用过高时应当不选择注资; 应当采取 Barrier 策略分红; 当破产终端值增大时破产的损害减小， 保险公司对破产的敏感性减小. 特别是当破产终端值足够大时, 保险公司应当立即宣告破产并获得终 端值; 与 “便宜” 再保险不同 (参见文献 [3,10]), 本文研究显示, 在 “不便宜” 再保险下不可以将所有业 务转移给再保险公司, 即风险自留水平必须大于 0 . 总之, 最优策略取决于模型参数之间的关系.

致谢 衰心感谢匿名审稿人提出的宝贵改进意见.

\section{参考文献}

1 De Finetti B. Su un'impostazione alternativa della teoria collettiva del rischio. In: Transactions of the XVth International Congress of Actuaries, vol. 2. New York: Congrès International d'Actuaires, 1957, 433-443

2 Asmussen S, H申gaard B, Taksar M. Optimal risk control and dividend distribution policies: Example of excess-of-loss reinsurance for an insurance corporation. Finance Stoch, 2000, 4: 299-324

3 Bai L, Guo J, Zhang H. Optimal excess-of-loss reinsurance and dividend payments with both transaction costs and taxes. Quant Finance, 2010, 10: 1163-1172

4 Cadenillas A, Choulli T, Taksar M, et al. Classical and impulse stochastic control for the optimization of the dividend and risk policies of an insurance firm. Math Finance, 2006, 16: 181-202

5 Chen M, Peng X, Guo J. Optimal dividend problem with a nonlinear regular-singular stochastic control. Insurance Math Econom, 2013, 52: 448-456

6 Choulli T, Taksar M, Zhou X. A diffusion model for optimal dividend distribution for a company with constrains on risk control. SIAM J Control Optim, 2003, 41: 1946-1979

7 H $\phi$ gaard B, Taksar M. Controlling risk exposure and dividends payout schemes: Insurance company example. Math Finance, 1999, 9: 153-182

$8 \mathrm{H} \phi$ gaard B, Taksar M. Optimal dynamic portfolio selection for a corporation with controllable risk and dividend distribution policy. Quant Finance, 2004, 4: 315-327

9 Taksar M. Dependence of the optimal risk control decisions on the terminal value for a financial corporation. Ann Oper Res, 2000, 98: 89-99

$10 \mathrm{Xu}$ J, Zhou M. Optimal risk control and dividend distribution policies for a diffusion model with terminal value. Math Comput Modelling, 2012, 56: 180-190

11 Taksar M, Hunderup C. The influence of bankruptcy value on optimal risk control for diffusion models with proportional reinsurance. Insurance Math Econom, 2007, 40: 311-321 
12 Yao D, Yang H, Wang R. Optimal risk and dividend control problem with fixed costs and salvage value: Variance premium principle. Econom Modelling, 2014, 37: 53-64

13 Liang Z, Young V R. Dividends and reinsurance under a penalty for ruin. Insurance Math Econom, 2012, 50: 437-445

14 Avram F, Palmowski Z, Pistorius M R. On the optimal dividend problem for a spectrally negative Lévy process. Ann Appl Probab, 2007, 17: 156-180

15 Kulenko N, Schmidli H. Optimal dividend strategies in a Cramér-Lundberg model with capital injections. Insurance Math Econom, 2008, 43: 270-278

$16 \mathrm{~L} \phi \mathrm{kka}$ A, Zervos M. Optimal dividend and issuance of equity policies in the presence of proportional costs. Insurance Math Econom, 2008, 42: 954-961

17 Paulsen J. Optimal dividend payments and reinvestments of diffusion processes with both fixed and proportional costs. SIAM J Control Optim, 2008, 47: 2201-2226

18 Sethi S, Taksar M. Optimal financing of a corporation subject to random returns. Math Finance, 2002, 12: 155-172

19 Yao D, Yang H, Wang R. Optimal dividend and capital injection problem in the dual model with proportional and fixed transaction costs. European J Oper Res, 2011, 211: 568-576

20 Guan H, Liang Z. Viscosity solution and impulse control of the diffusion model with reinsurance and fixed transaction costs. Insurance Math Econom, 2014, 54: 109-122

21 Meng H, Siu T. Optimal mixed impulse-equity insurance control problem with reinsurance. SIAM J Control Optim, 2011, 49: 254-279

22 Peng X, Chen M, Guo J. Optimal dividend and equity issuance problem with proportional and fixed transaction costs. Insurance Math Econom, 2012, 51: 576-585

23 Zhou M, Yuen K C. Optimal reinsurance and dividend for a diffusion model with capital injection: Variance premium principle. Econom Modelling, 2012, 29: 198-207

24 Grandell J. Aspects of Risk Theory. New York: Springer-Verlag, 1991

25 Fleming W, Soner H. Controlled Markov Process and Viscosity Solutions. New York: Springer-Verlag, 1993

\section{附录 相关证明}

\section{附录 A 定理 3.1 的证明}

(1) 对任意策略 $\pi=\left(a^{\pi} ; D^{\pi} ; R^{\pi}\right) \in \Pi$, 定义

$$
\Lambda_{D}^{\pi}=\left\{s: D_{s-}^{\pi} \neq D_{s}^{\pi}\right\}, \quad \Lambda_{R}^{\pi}=\left\{s: R_{s-}^{\pi} \neq R_{s}^{\pi}\right\}=\left\{\tau_{1}^{\pi}, \tau_{2}^{\pi}, \ldots, \tau_{i}^{\pi}, \ldots\right\}
$$

令 $\hat{D}_{t}^{\pi}=\sum_{s \in \Lambda_{D}^{\pi}, s \leqslant t}\left(D_{s}^{\pi}-D_{s-}^{\pi}\right)$ 为过程 $D_{t}^{\pi}$ 的跳跃部分, 而 $\tilde{D}_{t}^{\pi}=D_{t}^{\pi}-\hat{D}_{t}^{\pi}$ 表示过程 $D_{t}^{\pi}$ 的连续部分. 类似地, $\hat{R}_{t}^{\pi}$ 和 $\tilde{R}_{t}^{\pi}$ 分别表示过程 $R_{t}^{\pi}$ 的跳跃部分和连续部分. 利用 Itô 公式可得

$$
\begin{aligned}
\mathrm{e}^{-\delta\left(t \wedge T^{\pi}\right)} v\left(X_{t \wedge T^{\pi}}^{\pi}\right)-v(x) \\
=\int_{0}^{t \wedge T^{\pi}} \mathrm{e}^{-\delta s} \mathscr{A}^{a^{\pi}} v\left(X_{s}^{\pi}\right) d s+\int_{0}^{t \wedge T^{\pi}} \mathrm{e}^{-\delta s} \sqrt{\lambda \nu\left(a^{\pi}\left(X_{s}^{\pi}\right)\right)} v^{\prime}\left(X_{s}^{\pi}\right) d B_{s}-\int_{0}^{t \wedge T^{\pi}} \mathrm{e}^{-\delta s} v^{\prime}\left(X_{s}^{\pi}\right) d \tilde{D}_{s}^{\pi} \\
\quad+\int_{0}^{t \wedge T^{\pi}} \mathrm{e}^{-\delta s} v^{\prime}\left(X_{s}^{\pi}\right) d \tilde{R}_{s}^{\pi}+\sum_{s \in \Lambda_{R}^{\pi} \cup \Lambda_{D}^{\pi}, s \leqslant t \wedge T^{\pi}} \mathrm{e}^{-\delta s}\left(v\left(X_{s}^{\pi}\right)-v\left(X_{s-}^{\pi}\right)\right) .
\end{aligned}
$$

(A.1) 中右式的最后一项可以表示为

$$
\begin{aligned}
& \quad \sum_{s \in \Lambda_{R}^{\pi} \cup \Lambda_{D}^{\pi}, s \leqslant t \wedge T^{\pi}} \mathrm{e}^{-\delta s}\left(v\left(X_{s}^{\pi}\right)-v\left(X_{s-}^{\pi}\right)\right) \\
& =\sum_{s \in \Lambda_{D}^{\pi}, s \leqslant t \wedge T^{\pi}} \mathrm{e}^{-\delta s}\left(v\left(X_{s}^{\pi}\right)-v\left(X_{s-}^{\pi}\right)\right)+\sum_{s \in \Lambda_{R}^{\pi}, s \leqslant t \wedge T^{\pi}} \mathrm{e}^{-\delta s}\left(v\left(X_{s}^{\pi}\right)-v\left(X_{s-}^{\pi}\right)\right)
\end{aligned}
$$




$$
\leqslant-\sum_{s \in \Lambda_{D}^{\pi}, s \leqslant t \wedge T^{\pi}} \mathrm{e}^{-\delta s} \beta_{1}\left(D_{s}^{\pi}-D_{s-}^{\pi}\right)+\sum_{i=1}^{\infty} \mathrm{e}^{-\delta \tau_{i}^{\pi}}\left(\beta_{2} \eta_{i}^{\pi}+K\right) I_{\left\{\tau_{i}^{\pi} \leqslant t \wedge T^{\pi}\right\}} .
$$

$v(x)$ 满足方程 (3.1) 保证了 (A.2) 中不等式成立. 另外, 由 (3.1) 可知 (A.1) 右式的第一项是非正的. 因此, 将 (A.2) 代入 (A.1) 可得

$$
\begin{aligned}
\mathrm{e}^{-\delta\left(t \wedge T^{\pi}\right)} v\left(X_{t \wedge T^{\pi}}^{\pi}\right) \leqslant & v(x)+\int_{0}^{t \wedge T^{\pi}} \mathrm{e}^{-\delta s} \sqrt{\lambda \nu\left(a^{\pi}\left(X_{s}^{\pi}\right)\right)} v^{\prime}\left(X_{s}^{\pi}\right) d B_{s} \\
& -\beta_{1} \int_{0}^{t \wedge T^{\pi}} \mathrm{e}^{-\delta s} d D_{s}^{\pi}+\sum_{i=1}^{\infty} \mathrm{e}^{-\delta \tau_{i}^{\pi}}\left(\beta_{2} \eta_{i}^{\pi}+K\right) I_{\left\{\tau_{i}^{\pi} \leqslant t \wedge T^{\pi}\right\}} .
\end{aligned}
$$

注意到 $v^{\prime}(x)$ 是有界的, 所以, (A.3) 右式第二项是一致可积鞅. 那么, 对 (A.3) 两边求积分得

$$
v(x) \geqslant \mathrm{E}^{x}\left(\beta_{1} \int_{0}^{T^{\pi}} \mathrm{e}^{-\delta s} d D_{s}^{\pi}+P \mathrm{e}^{-\delta T^{\pi}}-\sum_{i=1}^{\infty} \mathrm{e}^{-\delta \tau_{i}^{\pi}}\left(\beta_{2} \eta_{i}^{\pi}+K\right) I_{\left\{\tau_{i}^{\pi} \leqslant T^{\pi}\right\}}\right)=V(x, \pi) .
$$

从而不等式 $v(x) \geqslant V(x, \pi)$ 成立.

(2) 更进一步, 如果存在策略 $\pi^{*} \in \Pi$ 使得 $v(x)=V\left(x, \pi^{*}\right)$, 那么, 值函数的最优性可推出

$$
v(x) \leqslant V(x)=\max _{\pi \in \Pi} V(x, \pi) .
$$

又因为 (1) 中已经证明了 $v(x) \geqslant V(x)$ 成立, 所以, $v(x)=V(x)=V\left(x, \pi^{*}\right)$.

\section{附录 B 不等式 (4.47) 的证明}

在 $\frac{2 m \mu_{m}}{\nu_{m}} \theta_{1} \leqslant \theta_{2} \leqslant \frac{m}{\lambda \nu_{m}}\left(\lambda \theta_{1} \mu_{m}+\sqrt{\left(\lambda \theta_{1} \mu_{m}\right)^{2}+2 \lambda \delta \nu_{m}}\right)=-m r_{-}$情形下, 容易验证 (4.47) 的左式 是负数, 从而不等式自然成立. 接下来讨论 $\theta_{1}<\theta_{2} \leqslant \frac{2 m \mu_{m}}{\nu_{m}} \theta_{1}$ 的情形. 考虑一个关于变量 $\theta_{2}$ 的函数

$$
\Psi\left(\theta_{2}\right)=\frac{m}{m+\frac{\theta_{2}}{r_{+}}}\left(\frac{m+\frac{\theta_{2}}{r_{+}}}{m+\frac{\theta_{2}}{r_{-}}}\right)^{\frac{-r_{-}}{r_{+}-r_{-}}}\left(\mu_{m} \theta_{1}-\frac{\nu_{m}}{2 m} \theta_{2}\right)-\mu_{m} \theta_{1}, \quad \theta_{2} \in\left[\theta_{1}, \frac{2 m \mu_{m}}{\nu_{m}} \theta_{1}\right] .
$$

根据 Viete 定理知,

$$
r_{+}+r_{-}=-\frac{2 \mu_{m} \theta_{1}}{\nu_{m}}, \quad r_{+} r_{-}=\frac{-2 \delta}{\lambda \nu_{m}}
$$

利用 (B.2) 可得

$$
\begin{aligned}
\frac{d}{d \theta_{2}} \log \Psi\left(\theta_{2}\right) & =\frac{\lambda \theta_{2} \nu_{m}}{2 \delta\left(m+\frac{\theta_{2}}{r_{+}}\right)\left(m+\frac{\theta_{2}}{r_{-}}\right)}-\frac{\nu_{m}}{2 m \nu_{m} \theta_{1}-\nu_{m} \theta_{2}} \\
& =\frac{\lambda \theta_{2} \nu_{m}}{2 \delta m^{2}+2 \lambda m \mu_{m} \theta_{1} \theta_{2}-\lambda \nu_{m} \theta_{2}^{2}}-\frac{\nu_{m}}{2 m \nu_{m} \theta_{1}-\nu_{m} \theta_{2}} \\
& =\frac{\nu_{m}}{\frac{2 \delta m^{2}}{\lambda \theta_{2}}+2 m \mu_{m} \theta_{1}-\nu_{m} \theta_{2}}-\frac{\nu_{m}}{2 m \nu_{m} \theta_{1}-\nu_{m} \theta_{2}}<0 .
\end{aligned}
$$

因此, $\Psi\left(\theta_{2}\right)$ 在区间 $\left[\theta_{1}, \frac{2 m \mu_{m}}{\nu_{m}} \theta_{1}\right]$ 上递减. 考虑到 $\nu_{m} \leqslant 2 m \mu_{m}$ 和 $r_{-}<-\frac{2 \mu_{m} \theta_{1}}{\nu_{m}}$, 我们可计算得到

$$
\Psi\left(\theta_{2}\right) \leqslant \Psi\left(\theta_{1}\right)=\frac{m}{m+\frac{\theta_{1}}{r_{+}}}\left(\frac{m+\frac{\theta_{1}}{r_{+}}}{m+\frac{\theta_{1}}{r_{-}}}\right)^{\frac{-r_{-}}{r_{+}-r_{-}}}\left(\mu_{m} \theta_{1}-\frac{\nu_{m}}{2 m} \theta_{1}\right)-\mu_{m} \theta_{1}
$$




$$
\begin{aligned}
& <\frac{m}{m+\frac{\theta_{1}}{r_{+}}}\left(\frac{m+\frac{\theta_{1}}{r_{+}}}{m+\frac{\theta_{1}}{r_{-}}}\right)\left(\mu_{m} \theta_{1}-\frac{\nu_{m}}{2 m} \theta_{1}\right)-\mu_{m} \theta_{1} \\
& =\frac{m \theta_{1}}{m+\frac{\theta_{1}}{r_{-}}}\left(\mu_{m}-\frac{\nu_{m}}{2 m}\right)-\mu_{m} \theta_{1} \\
& <\frac{m \theta_{1}}{m+\frac{\theta_{1}}{-\frac{2 \mu_{m} \theta_{1}}{\nu_{m}}}}\left(\mu_{m}-\frac{\nu_{m}}{2 m}\right)-\mu_{m} \theta_{1}=0 .
\end{aligned}
$$

总之, (4.47) 对任意 $\theta_{2} \in\left(\theta_{1}, \frac{m}{\lambda \nu_{m}}\left(\lambda \theta_{1} \mu_{m}+\sqrt{\left(\lambda \theta_{1} \mu_{m}\right)^{2}+2 \lambda \delta \nu_{m}}\right)\right)$ 也成立, 结论得证.

\section{附录 C 引理 $4.2(2)$ 的证明}

显然, 引理 4.2(2) 中 $f(x)$ 是一个二阶连续可导且递增的凹函数, 其导函数满足 $\beta_{1} \leqslant f^{\prime}(x) \leqslant f^{\prime}(0)$ $<\infty$. 接下来验证 $f(x)$ 和 $a^{\pi^{*}}(x)$ 满足方程 (3.1) 和 (3.2).

第 1 步 证明 $\mathscr{M} f(x)-f(x) \leqslant 0$. 我们有

$$
\begin{aligned}
\mathscr{M} f(x)-f(x) & =\max _{y \geqslant 0}\left\{f(x+y)-\beta_{2} y-K\right\}-f(x) \\
& =\max _{y \geqslant 0}\left\{\int_{x}^{x+y}\left(f^{\prime}(s)-\beta_{2}\right) d s\right\}-K .
\end{aligned}
$$

(i) 如果 $0 \leqslant x \leqslant \xi_{1}^{*}$, 那么 $f^{\prime}(x)-\beta_{2} \geqslant 0$ 成立当且仅当 $0 \leqslant x \leqslant \xi_{1}^{*}$. 因此,

$$
\begin{aligned}
\mathscr{M} f(x)-f(x) & =\max _{y \geqslant 0}\left\{\int_{x}^{x+y}\left(f^{\prime}(s)-\beta_{2}\right) d s\right\}-K \\
& \leqslant \int_{0}^{\xi_{1}^{*}}\left(f^{\prime}(s)-\beta_{2}\right) d s-K=I_{1}\left(\xi_{1}^{*}\right)-K \leqslant 0 .
\end{aligned}
$$

(ii) 如果 $\xi_{1}^{*}<x<\infty$, 则有 $f^{\prime}(x)-\beta_{2}<0$ 成立. 可计算

$$
\mathscr{M} f(x)-f(x)=\max _{y \geqslant 0}\left\{\int_{x}^{x+y}\left(f^{\prime}(s)-\beta_{2}\right) d s\right\}-K=-K<0 .
$$

第 2 步 证明 $\max _{0 \leqslant a \leqslant m}\left\{\mathscr{A}^{a} f(x)\right\} \leqslant 0$ 对任意 $x \geqslant 0$ 成立.

(i) 如果 $0 \leqslant x \leqslant b_{0}$, 已知 $f(x)$ 和 $a^{\pi^{*}}(x)$ 满足 (4.2), 即 $\max _{0 \leqslant a \leqslant m}\left\{\mathscr{A}^{a} f(x)\right\}=\mathscr{A}^{a^{\pi^{*}}} f(x)=0$.

(ii) 如果 $x>b_{0}$, 那么, $f(x) \geqslant f\left(b_{0}\right), f^{\prime}(x)=f^{\prime}\left(b_{0}\right)=\beta_{1}$ 且 $f^{\prime \prime}(x)=f^{\prime \prime}\left(b_{0}\right)=0$. 所以, 对任意 $a \in[0, m]$ 可知,

$$
\begin{aligned}
\mathscr{A}^{a} f(x) & =\frac{1}{2} \lambda \nu(a) f^{\prime \prime}(x)+\left[\left(\theta_{1}-\theta_{2}\right) \lambda \mu_{m}+\theta_{2} \lambda \mu(a)\right] f^{\prime}(x)-\delta f(x) \\
& =\frac{1}{2} \lambda \nu(a) f^{\prime \prime}\left(b_{0}\right)+\left[\left(\theta_{1}-\theta_{2}\right) \lambda \mu_{m}+\theta_{2} \lambda \mu(a)\right] f^{\prime}\left(b_{0}\right)-\delta f(x) \\
& \leqslant \frac{1}{2} \lambda \nu(a) f^{\prime \prime}\left(b_{0}\right)+\left[\left(\theta_{1}-\theta_{2}\right) \lambda \mu_{m}+\theta_{2} \lambda \mu(a)\right] f^{\prime}\left(b_{0}\right)-\delta f\left(b_{0}\right) \\
& =\mathscr{A}^{a} f\left(b_{0}\right) \leqslant 0 .
\end{aligned}
$$

第 3 步 证明 $\beta_{1}-f^{\prime}(x) \leqslant 0$.

从 (4.30) 中 $f(x)$ 的表达式可知该不等式显然成立. 
第 4 步 证明 $\max \left\{\mathscr{M} f(x)-f(x), \max _{0 \leqslant a \leqslant m}\left\{\mathscr{A}^{a} f(x)\right\}, \beta_{1}-f^{\prime}(x)\right\}=0$.

(i) 如果 $x=0$, 则有 $\mathscr{M} f(0)-f(0) \leqslant 0$;

(ii) 如果 $0 \leqslant x \leqslant b_{0}$, 则有 $\max _{0 \leqslant a \leqslant m}\left\{\mathscr{A}^{a} f(x)\right\}=0$;

(iii) 如果 $x>b_{0}$, 则有 $\beta_{1}-f^{\prime}(x)=0$.

第 5 步 显然, $\mathscr{M} f(0)-f(0)<0$ 和 $P-f(0)=0$ 成立.

综上所述, $f(x)$ 和 $a^{\pi^{*}}$ 满足 (3.1) 和 (3.2).

\section{附录 D 定理 4.1 中情形 7 的证明}

令 $\tilde{f}_{1}(x)$ 如 (4.55) 所示, 策略 $\pi^{*}=\left(\tilde{a}_{1}^{\pi^{*}}, D^{\pi^{*}}, R^{\pi^{*}}\right) \in \Pi$ 由 (4.56)、(4.70) (参数取 $\left.b=b_{1}\right) 、(4.73)$ 和 (4.74) 给定. 由 (4.51) 可知, 对 $0 \leqslant X_{s}^{\pi^{*}} \leqslant b_{1}$ 有 $\mathscr{A}^{\tilde{a}_{1}^{\pi^{*}}} \tilde{f}_{1}\left(X_{s}^{\pi^{*}}\right)=0$ 成立. 对任意 $t>0$, 有

$$
\int_{0}^{t \wedge T^{\pi^{*}}} \mathrm{e}^{-\delta s} \mathscr{A}^{\tilde{a}_{1}^{* *}} \tilde{f}_{1}\left(X_{s}^{\pi^{*}}\right) d s=\int_{0}^{t \wedge T^{\pi^{*}}} \mathrm{e}^{-\delta s} \mathscr{A}^{\tilde{a}_{1}^{* *}} \tilde{f}_{1}\left(X_{s}^{\pi^{*}}\right) I_{\left\{0 \leqslant X_{s}^{\pi^{*}} \leqslant b_{1}\right\}} d s=0
$$

成立. 另外 (4.70)、(4.73) 和 (4.74) 可推出

$$
\begin{aligned}
& \sum_{s \in \Lambda_{R}^{\pi^{*}} \cup \Lambda_{D}^{\pi^{*}}, s \leqslant t \wedge T^{\pi^{*}}} \mathrm{e}^{-\delta s}\left(\tilde{f}_{1}\left(X_{s}^{\pi^{*}}\right)-\tilde{f}_{1}\left(X_{s-}^{\pi^{*}}\right)\right) \\
= & \sum_{s \in \Lambda_{D}^{\pi^{*}}, s \leqslant t \wedge T^{\pi^{*}}} \mathrm{e}^{-\delta s}\left(\tilde{f}_{1}\left(X_{s}^{\pi^{*}}\right)-\tilde{f}_{1}\left(X_{s-}^{\pi^{*}}\right)\right) I_{\left\{X_{s}^{\pi^{*}}=b_{1}\right\}} \\
& +\sum_{s \in \Lambda_{R}^{\pi^{*}}, s \leqslant t \wedge T \pi^{\pi^{*}}} \mathrm{e}^{-\delta s}\left(\tilde{f}_{1}\left(X_{s}^{\pi^{*}}\right)-\tilde{f}_{1}\left(X_{s-}^{\pi^{*}}\right)\right) I_{\left\{X_{s}^{\pi^{*}}=0\right\}} \\
= & -\sum_{s \in \Lambda_{D}^{\pi^{*}}, s \leqslant t \wedge T \pi^{\pi^{*}}} \mathrm{e}^{-\delta s} \beta_{1}\left(D_{s}^{\pi^{*}}-D_{s-}^{\pi^{*}}\right)+\sum_{i=1}^{\infty} \mathrm{e}^{-\delta \tau_{i}^{\pi^{*}}}\left(\beta_{2} \eta_{i}^{\pi^{*}}+K\right) I_{\left\{\tau_{i}^{\pi^{*}} \leqslant t \wedge T^{\pi^{*}}\right\}} .
\end{aligned}
$$

用 $\pi^{*} 、 T^{\pi^{*}}=\infty$ 和 $\tilde{f}_{1}$ 分别替换 (A.1) 中 $\pi 、 T^{\pi}$ 和 $v$, 并对两边求期望可得

$$
\begin{aligned}
\tilde{f}_{1}(x) & =\mathrm{E}^{x}\left(\beta_{1} \int_{0}^{T^{\pi^{*}}} \mathrm{e}^{-\delta s} d D_{s}^{\pi^{*}}+P \mathrm{e}^{-\delta T^{\pi^{*}}}-\sum_{i=1}^{\infty} \mathrm{e}^{-\delta \tau_{i}^{\pi^{*}}}\left(\beta_{2} \eta_{i}^{\pi^{*}}+K\right) I_{\left\{\tau_{i}^{\pi^{*}} \leqslant T^{\pi^{*}}\right\}}\right) \\
& =\mathrm{E}^{x}\left(\beta_{1} \int_{0}^{T^{\pi^{*}}} \mathrm{e}^{-\delta s} d D_{s}^{\pi^{*}}-\sum_{i=1}^{\infty} \mathrm{e}^{-\delta \tau_{i}^{\pi^{*}}}\left(\beta_{2} \eta_{i}^{\pi^{*}}+K\right) I_{\left\{\tau_{i}^{\pi^{*}} \leqslant T^{\left.\pi^{*}\right\}}\right.}\right) \\
& =V\left(x, \pi^{*}\right) .
\end{aligned}
$$

另外, 根据定理 3.1 可知 $\tilde{f}_{1}(x) \geqslant V(x)=\max _{\pi \in \Pi} V(x, \pi)$ 成立. 从而得到 $\tilde{f}_{1}(x)=V(x)=V\left(x, \pi^{*}\right)$.

\section{Optimal dividend, capital injection and excess-of-loss reinsurance strategies for insurer with a terminal value of the bankruptcy}

YAO DingJun, WANG RongMing \& XU Lin

Abstract In this paper, we assume that an insurance company can control dividend, capital injection and excess-of-loss reinsurance strategies dynamically. Transaction costs are incurred by these business activities: 
Reinsurance is "non-cheap", dividend is taxed, reinvestment generates proportional and fixed costs. An arbitrary terminal value of the bankruptcy is considered. Under the objective of maximizing the company's value, we present the optimal strategies and analyze the effects of transaction costs and terminal value. The results show that capital injection maybe profitable if transaction costs and terminal value are relatively low, dividends should be paid under the barrier strategy, less reinsurance is bought when the surplus increases.

Keywords dividend, capital injection, excess-of-loss reinsurance, terminal value, transaction cost, optimal strategy

$\operatorname{MSC}(2010) \quad 62 \mathrm{P} 05,97 \mathrm{M} 30,93 \mathrm{E} 20$

doi: 10.1360/SCM-2014-0564 